\title{
Enteropathogenic E. coli disrupts tight junction barrier function and structure in vivo
}

\author{
Donnie E Shifflett ${ }^{1}$, Daniel R Clayburgh ${ }^{2}$, Athanasia Koutsouris ${ }^{1}$, Jerrold R Turner ${ }^{2, *}$ \\ and Gail A Hecht ${ }^{1, *}$ \\ ${ }^{1}$ Department of Medicine, Section of Digestive Diseases and Nutrition, University of Illinois at Chicago, \\ Chicago, IL, USA and ${ }^{2}$ Department of Pathology, The University of Chicago, Chicago, IL, USA
}

\begin{abstract}
Enteropathogenic Escherichia coli (EPEC) infection disrupts tight junctions (TJs) and perturbs intestinal barrier function in vitro. $E$. coli secreted protein $F(E s p F)$ is, in large part, responsible for these physiological and morphological alterations. We recently reported that the C57BL/6J mouse is a valid in vivo model of EPEC infection as EPEC colonizes the intestinal epithelium and effaces microvilli. Our current aim was to examine the effects of EPEC on TJ structure and barrier function of the mouse intestine and to determine the role of EspF in vivo. C57BL/6J mice were gavaged with $\sim 2 \times 10^{8}$ EPEC organisms or PBS. At 1 or 5 days postinfection, mice were killed and ileal and colonic tissue was mounted in Üssing chambers to determine barrier function (measured as transepithelial resistance) and short circuit current. TJ structure was analyzed by immunofluorescence microscopy. Wild-type (WT) EPEC significantly diminished the barrier function of ileal and colonic mucosa at 1 and 5 days postinfection. Deficits in barrier function correlated with redistribution of occludin in both tissues. Infection with an EPEC strain deficient of EspF ( $\triangle e s p F)$ had no effect on barrier function at 1 day postinfection. Furthermore, $\Delta e s p F$ had no effect on ileal TJ morphology and minor alterations of colonic TJ morphology at 1 day postinfection. In contrast, at 5 days postinfection, WT EPEC and $\triangle E s p F$ had similar effects on barrier function and occludin localization. In both cases this was associated with immune activation, as demonstrated by increased mucosal tumor necrosis factor- $\alpha$ levels 5 days postinfection. In conclusion, these data demonstrate that WT EPEC infection of 6-8-week-old C57BL/6J mice (1) significantly decreases barrier function in the ileum and colon (2) redistributes occludin in the ileum and colon and (3) is dependent upon EspF to induce TJ barrier defects at early, but not late, times postinfection.
\end{abstract}

Laboratory Investigation (2005) 85, 1308-1324. doi:10.1038/labinvest.3700330; published online 15 August 2005

Keywords: enteric pathogen; EPEC; EspF; Occludin; TNF- $\alpha$; ZO-1

Enteropathogenic Escherichia coli (EPEC) induces characteristic attaching and effacing (A/E) lesions in host intestinal epithelial cells through intimate attachment to the apical epithelial surface and subsequent recruitment of cytoskeletal proteins., ${ }^{1,2}$ The EPEC type III secretion system (TTSS) allows numerous effector molecules to be injected into host intestinal epithelial cells. The EPEC genes that confer virulence reside on a $35.6 \mathrm{kB}$ pathogenicity island known as the locus of enterocyte effacement (LEE). ${ }^{3}$ The LEE encodes proteins for the TTSS, adherence factors, chaperones, and effector molecules. The outer membrane protein, intimin, and the TTSS-dependent-translocated intimin receptor, are

Correspondence: Dr GA Hecht, MD, Department of Medicine, Section of Digestive Diseases and Nutrition, University of Illinois at Chicago, Chicago, IL 60612, USA.

E-mail: gahecht@uic.edu

*Designates cosenior authors.

Received 12 May 2005; revised and accepted 6 July 2005; published online 15 August 2005 responsible for intimate adherence of EPEC to intestinal epithelial cells. Additionally, secreted proteins such as EspF, EspG, EspH, and Map, are regarded as effector molecules that contribute to EPEC pathogenesis ${ }^{4,5}$ and are involved in perturbing the physiological functions of host intestinal epithelial cells. EPEC-induced physiological alterations include inflammation, ${ }^{6,7}$ disruption in transport processes, ${ }^{8-10}$ and perturbation of intestinal barrier function. ${ }^{11-17}$

Recent in vitro studies have demonstrated that the EPEC effector protein EspF plays a central role in decreasing transepithelial resistance (TER) and altering the intestinal epithelial tight junction (TJ) structure. ${ }^{11,13,18,19}$ Specifically, EPEC induces disruption of TJ architecture as evidenced by a loss of TJ protein-protein interactions, redistribution of TJ proteins, and the appearance of aberrant $\mathrm{TJ}$ strands in the lateral membrane. ${ }^{12}$ Although the mechanism by which EspF perturbs intestinal barrier function in vitro has not been defined, it is known that strains deficient in $e s p F$ are not attenuated in their ability to 
form characteristic A/E lesions. ${ }^{11,20}$ Thus, the observed physiological effects of EspF are not due to a defect in EPEC attachment to host cells.

The majority of published studies regarding EPEC pathogenesis have utilized in vitro models primarily due to the lack of representative small animal models. As a result, much of our understanding of EPEC infection in vivo has been extrapolated from studies of rabbit enteropathogenic E. coli (REPEC) and the murine pathogen Citrobacter rodentium. While these organisms are similar to the human pathogen EPEC and have yielded insightful data regarding in vivo pathophysiology, ${ }^{21-25}$ limitations associated with these animal models include restricted genetic and immunological resources and pathophysiological differences. ${ }^{21}$ For example, $C$. rodentium induces mucosal hyperplasia and attaches via a primary adhesion. ${ }^{25}$ Until recently, there was no established small animal model for human EPEC infection. Our laboratory, however, recently characterized the $\mathrm{C} 57 \mathrm{BL} / 6 \mathrm{~J}$ mouse as a model of EPEC infection by demonstrating that this human pathogen colonizes the intestinal mucosa, causes microvillous effacement, and triggers intestinal inflammation. ${ }^{26}$ Therefore, the aim of this study was to examine the effects of EPEC on the TJ structure and barrier function of the C57BL/6J mouse intestine and to determine the role of EspF in this in vivo model.

\section{Materials and methods}

\section{Infection}

Male, C57BL/6J mice (6 weeks old) were obtained from Jackson Laboratory. All animal procedures were approved by the University of Illinois-Chicago Animal Care and Use Committee. Mice were allowed to equilibrate in the Biological Resources Laboratory animal housing facility at the University of Illinois-Chicago for approximately 7 days. Following this equilibration period, mice were gavaged with $200 \mu \mathrm{l}$ of sterile PBS (control, uninfected) or $\sim 2 \times 10^{8}$ EPEC organisms suspended in $200 \mu \mathrm{l}$ sterile PBS utilizing a $4 \mathrm{~cm}$ long, curved needle with a steel ball at the tip.

\section{Electrophysiology}

At 1 or 5 days after infection, mice were killed via $\mathrm{CO}_{2}$ asphyxiation and intestinal tissue was resected. One section each of ileal and colonic tissue was mounted in an Üssing chamber for measurements of TER, an indicator of barrier function, and short circuit current $\left(I_{\mathrm{sc}}\right)$. Full thickness, unstripped muscosa was bathed on both mucosal and serosal surfaces with $5 \mathrm{ml}$ of oxygenated $\left(95 \% \mathrm{O}_{2} / 5 \% \mathrm{CO}_{2}\right)$ Ringer's solution containing (in $\mathrm{mM}$ ): $\mathrm{NaCl} 109.8$, $\mathrm{KCl} 5.3, \mathrm{CaCl}_{2} 1.2, \mathrm{MgCl}_{2} 1.2, \mathrm{NaHCO}_{3} 25, \mathrm{Na}_{2} \mathrm{HPO}_{4}$ 2.4, $\mathrm{NaH}_{2} \mathrm{PO}_{4}$ 0.4. In addition, the serosal bathing solution contained $10 \mathrm{mM}$ of glucose, which was osmotically balanced by $10 \mathrm{mM}$ mannitol on the mucosal side. Bathing solutions were circulated in water-jacketed reservoirs and maintained at $37^{\circ} \mathrm{C}$.

\section{Immunofluorescence}

Sections of ileal and colonic tissue from all mice killed for Üssing chamber analysis were snap-frozen in optimal cutting temperature embedding medium (Tissue-Tek O.C.T compound, Sakura Finetek USA, Inc., Torrance, CA, USA) and stored at $-80^{\circ} \mathrm{C}$. At least six sections from each of two to three animals for each condition were analyzed by immunofluorescence microscopy. Representative images are shown. For immunostaining, $4 \mu \mathrm{m}$ frozen sections were fixed with 1\% paraformaldehyde in PBS for $10 \mathrm{~min}$ at room temperature. After washing in PBS, permeabilization with $0.5 \%$ NP-40, and blocking of nonspecific binding sites with 5\% normal goat serum (NGS), tissues were incubated with monoclonal mouse antioccludin or rabbit anti-ZO-1 (both used at $0.5 \mu \mathrm{g} / \mathrm{ml}$, Zymed) in PBS with $1 \%$ NGS for $90 \mathrm{~min}$ at room temperature. After washing, sections were incubated with Alexa 594-conjugated goat anti-mouse IgG or goat anti-rabbit IgG antisera, respectively ( $8 \mu \mathrm{g} / \mathrm{ml}$ Molecular Probes), Alexa 488conjugated phalloidin (5 U/ml, Molecular Probes), and Hoechst 33342 (Molecular Probes) for $60 \mathrm{~min}$. Sections were then washed and mounted under coverslips using ProLong Gold antifade reagent (Molecular Probes). Sections were imaged using a Leica DM4000 epifluorescence microscope equipped with appropriate filter cubes (Chroma Technology, Brattleboro, VT, USA) and a Coolsnap HQ camera (Roper Scientific) controlled by MetaMorph 6 (Universal Imaging Corporation).

\section{Attachment Assays}

To determine the level of attachment of wild-type (WT) EPEC and genetically altered EPEC strains to both the ileum and the colon of C57BL/6J mice, attachment assays were performed. Tissue samples from the ileum and the colon were homogenized in sterile PBS plus $1 \%$ NP-40. Aliquots of $100 \mu \mathrm{l}$ were plated onto agar plates containing ampicillin or kanamycin. Ampicillin plates were used for WT EPEC that is Amp resistant due to an ampicillinresistant gene inserted on the pMar plasmid. Agar plates containing kanamycin were used for $\Delta e s p F$ infected mice as $\Delta e s p F$ is kanamycin resistant. Colony-forming units were counted and represented adherent bacteria.

\section{Gel Electrophoresis and Western Blotting}

Ileal and colonic tissue samples were collected immediately upon euthanasia of mice and snap- 
frozen in liquid nitrogen. In preparation for SDSPAGE, tissues were thawed to $4^{\circ} \mathrm{C}$. Tissue samples were homogenized in chilled RIPA buffer $(150 \mathrm{mM}$ $\mathrm{NaCl}, 50 \mathrm{mM}$ Tris-HCl, $\mathrm{pH} 7.4, \quad 0.5 \%$ sodium deoxycholate, $1 \%$ Triton X-100, $1 \mathrm{mM}$ EDTA), including protease and phosphotase inhibitors ( $1 \mathrm{mM}$ PMSF, $1 \mathrm{mM} \mathrm{Na} \mathrm{VO}_{4}, 1 \mathrm{mM} \mathrm{NaF}$, and $5 \mu \mathrm{g} /$ $\mathrm{ml}$ of each of aprotinin, leupeptin, pepstatin). After centrifugation at $10000 \mathrm{~g}$ for $10 \mathrm{~min}$ at $4^{\circ} \mathrm{C}$, the supernatant was recovered and assayed for protein content (DC protein assay; Bio-Rad, Hercules, CA, USA). Equal amounts of total protein were separated on $10 \%$ SDS-polyacrylamide gels and then transferred to a nitrocellulose membrane. After blocking overnight in Tris-buffered saline (TBS) containing $0.05 \%$ Tween (TBS-T) and 5\% dry powdered milk, membranes were washed three times for 5 min each with TBS-T and incubated for $2 \mathrm{~h}$ at room temperature in primary antibody (rabbit antioccludin, or rabbit anti-ZO-1, both from Zymed or rabbit antiactin, Sigma). After three washes with TBS-T, the membranes were incubated for $1 \mathrm{~h}$ with horseradish peroxidase-conjugated secondary antibody. Following two washes with TBS-T and one wash with TBS, the membranes were developed for visualization of protein by the addition of enhanced chemiluminescence reagent (Amersham, Princeton, NJ, USA). Densitometric analysis was performed (Alpha Imager 1220 system) on three individual mice per treatment group.

\section{Quantitative Real-Time RT-PCR}

Extracted ileal and colonic tissues $\left(\sim 3 \mathrm{~mm}^{3}\right)$ were immediately placed in Trizol. After brief sonication to disrupt the tissue, RNA was extracted with chloroform, precipitated with isopropanol, and resuspended in DEPC-treated water. RNA purification was performed utilizing an RNeasy mini kit (Qiagen). The generation of cDNA was completed from $2 \mu \mathrm{g}$ of RNA using Thermoscript reverse transcriptase (Invitrogen) and random hexamer primers in a $25 \mu \mathrm{l}$ reaction. The mRNA levels of tumor necrosis factor- $\alpha$ (TNF- $\alpha$ ) were determined by SYBR green real-time PCR using the MyiQ Realtime PCR Detection System (BioRad) through 50 cycles. GAPDH was utilized as a reference. The TNF- $\alpha$ primers were TCAAGTGGCATAGATGTGGAAGAA and TGGCTCTGCAGGATTTTCATG and the GAPDH primers were CTTCACCACCATGGAGAAGGC and GGCATGGACTGTGGTCATGAG. Data were standardized to GAPDH for each sample and then normalized to uninfected, control tissue sections. Three separate mice were analyzed per treatment group.

\section{Data Analysis}

All values are reported as mean \pm s.e. of the mean. Data from multiple treatment groups were compared utilizing a one-way analysis of variance with a post hoc Tukey's test. A ' $P$ '-value of $<0.05$ was considered statistically significant.

\section{Results}

\section{Adherence of WT EPEC and $\triangle e s p F$ In Vivo}

EPEC has been reported to colonize the intestine of the C57BL/6J mouse, induce inflammation and alter stool consistency after 10 days of infection. ${ }^{26}$ We therefore performed initial studies to determine whether EPEC colonizes the mouse intestine at 1 day postinfection as well as to determine if the mutant EPEC strain, $\triangle e s p F$, colonizes the murine intestine to a similar degree. Mice were infected with either WT EPEC or $\Delta e s p F$ for 1 or 5 days. Upon killing, sections of ileum and colon were extracted, weighed, homogenized, and plated to assess the attachment of the two EPEC strains. As expected, EPEC was not detected in the ileum or the colon of control, uninfected mice. Table 1 shows that EPEC colonization of the ileum and the colon occurred as early as 1 day postinfection and persisted for up to 5 days. Furthermore, there was no significant difference in the level of colonization by WT EPEC and $\Delta e s p F$ at day 1 or 5 in either tissue type.

\section{EPEC Disrupts Intestinal Epithelial Barrier Function at 1 Day Postinfection}

To determine if intestinal barrier function was perturbed by acute EPEC infection, as has been demonstrated in vitro, mice were infected with $2 \times 10^{8}$ EPEC organisms and killed 1 day postinfection. Segments of ileum and colon were mounted in Üssing chambers and electrophysiologic parameters were measured. As shown in Figure 1, WT EPEC significantly reduced the barrier function of the ileum (Figure 1a) and the colon (Figure 1b). Prior in vitro data have identified EspF as a key effector molecule in EPEC-induced alteration of TJ barrier function. ${ }^{11,18}$ Thus, we examined the role of EspF in the observed in vivo intestinal barrier disruption. In contrast to WT EPEC, infection with $\Delta e s p F$ had no effect on intestinal barrier function of either the ileum (Figure 1a) or the colon (Figure 1b) following 1 day of infection. Additionally, there were no differences in $I_{\mathrm{sc}}$ of ileal or colonic tissues (Figure 2)

Table 1 WT EPEC and $\Delta e s p F$ adherence in the ileum and the colon of C57BL/6J mice at 1 and 5 days postinfection

\begin{tabular}{lcc}
\hline & Ileum $\left(C F U \times 10^{3} / g\right)$ & Colon $\left(C F U \times 10^{3} / g\right)$ \\
\hline WT EPEC 1 day & $11.3 \pm 2.5$ & $8.5 \pm 1.7$ \\
WT EPEC 5 day & $12.9 \pm 2.8$ & $10.1 \pm 1.3$ \\
$\Delta e s p F$ 1 day & $15.6 \pm 1.1$ & $9.7 \pm 1.4$ \\
$\Delta e s p F$ 5 day & $11.0 \pm 1.2$ & $10.8 \pm 2.0$ \\
\hline
\end{tabular}

Values are means \pm s.e. of the mean ( $n=3$ per group). 

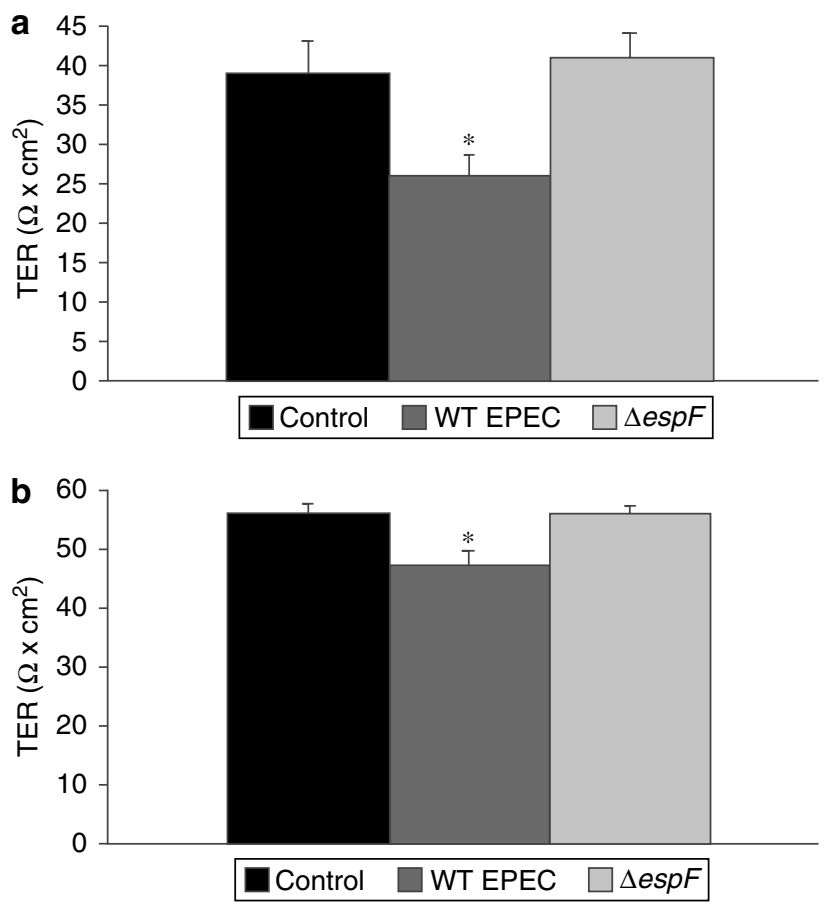

Figure 1 WT EPEC, but not $\triangle e s p F$, reduced intestinal barrier function at 1 day postinfection in C57BL/6J mice. (a) Segments of ileal tissue were mounted in Üssing chambers for assessment of transepithelial resistance (TER). WT EPEC induced a significant decrease in TER while mice infected with $\triangle e s p F$ have no alterations in TER when compared to uninfected control mice. (b) TER was also measured in excised colon. WT EPEC significantly diminished TER while mice infected with $\Delta e s p F$ have no alterations in TER when compared to uninfected, control mice. ${ }^{*} P<0.05$ when compared to control and $\Delta e s p F$. WT EPEC $(n=6), \Delta \operatorname{espF}(n=6)$, and control $(n=4)$. from control (uninfected), WT EPEC-infected, or $\Delta e s p F$-infected mice. This suggested that the decreased barrier function observed in WT EPECinfected mice requires EspF and is not a consequence of enhanced transcellular conductance.

\section{Epithelial Occludin Localization is Modified within 1 Day of WT EPEC Infection}

The aforementioned decrease in barrier function in both the ileum and the colon following a 1 day

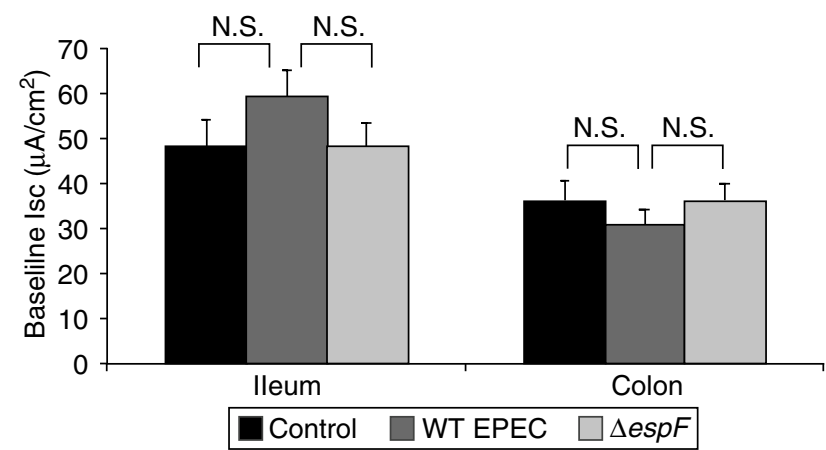

Figure 2 At 1 day of infection WT EPEC or $\triangle e s p F$ does not alter short circuit current $\left(I_{\mathrm{sc}}\right)$ in ileal or colonic tissue of C57BL/6] mice. Portions of ileum and colon were mounted in Üssing chambers as above. Ileal $I_{\mathrm{sc}}$ was similar in control tissue and tissue from mice infected with WT EPEC or $\Delta e s p F$ for 1 day. Similarly, WT EPEC and $\Delta e s p F$ did not alter colonic $I_{\mathrm{sc}}$ at 1 day postinfection. NS: not significant, $P>0.05$ for all comparisons; WT EPEC $(n=6), \Delta e s p F(n=6)$ and control $(n=4)$ lleum
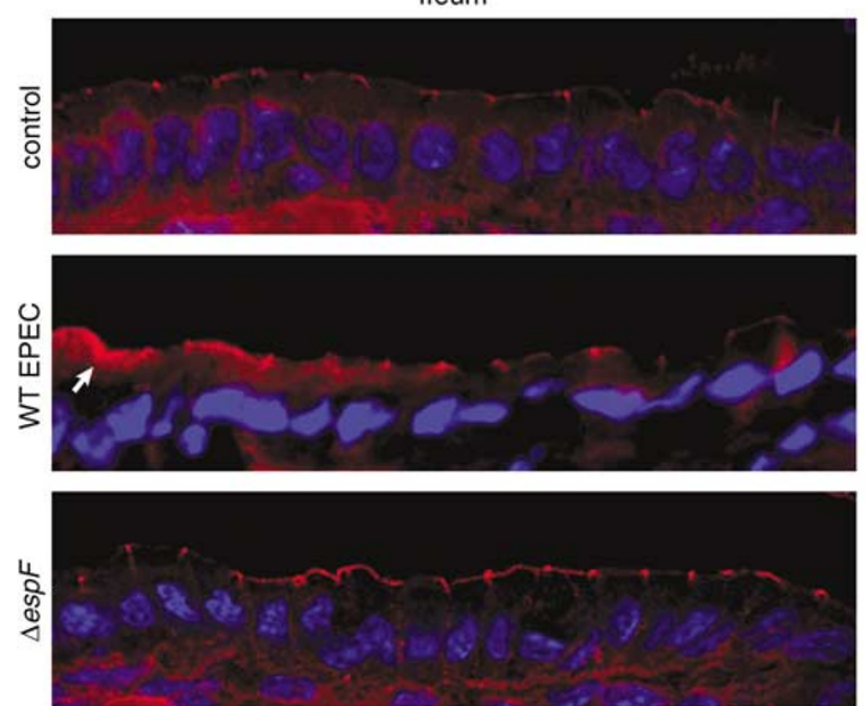

Colon
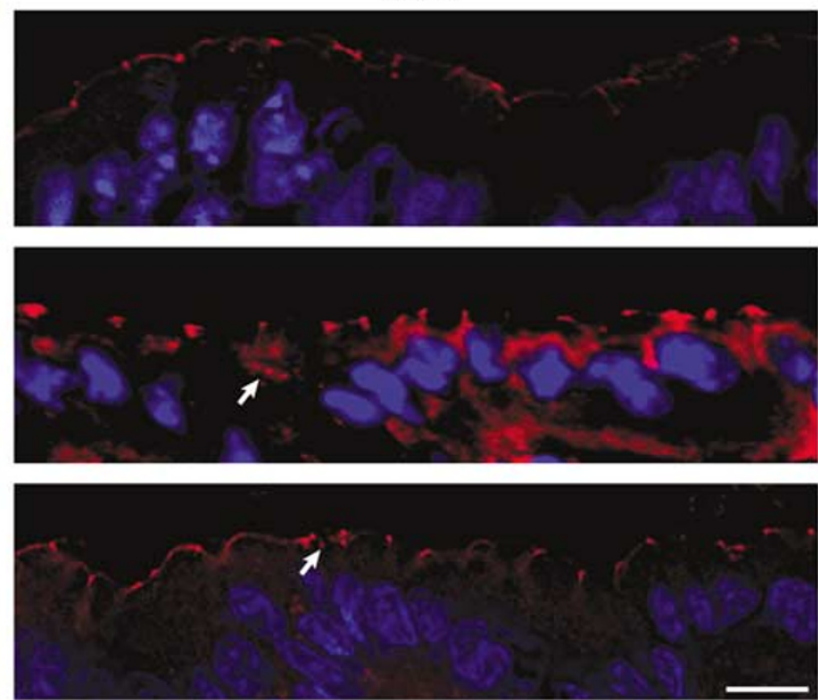

Figure 3 WT EPEC-infection causes redistribution of occludin in ileal and colonic epithelium 1 day postinfection. Frozen sections of ileum and colon were labeled for occludin (red), f-actin (green), and nuclei (blue). These low magnification views show that occludin is precisely localized to the tight junction in ileal and colonic epithelium from control uninfected mice. In contrast, both ileum and colon from WT EPEC-infected mice showed redistribution of occludin to include a cytoplasmic pool (see Figure 4) in approximately half of ileal epithelial cells and nearly all colonic epithelial cells. The distribution of occludin in ileal and colonic epithelium from $\Delta e s p F-$ infected mice was indistinguishable from that of control mice. Scale bar $=10 \mu \mathrm{m}$. 
infection with WT EPEC led us to determine if alterations in TJ structure accompanied this physiological change. In the ileum and the colon of control uninfected mice, the transmembrane protein occludin was precisely localized to the epithelial TJ. In well-oriented sections this is easily appreciated at low magnification as a regular series of bright red spots at the apical aspect of cell junctions (Figure 3). Examination at higher magnification revealed occludin colocalization with the apical perijunctional actomyosin ring in both ileal (Figure 4a) and colonic (Figure 4b) epithelia of control, uninfected mice. In contrast, occludin was present at the $\mathrm{TJ}$ as well as within the apical and basal cytoplasm of ileal and colonic epithelia of mice infected with WT EPEC for 1 day (Figure 3). At low magnification this is seen as both diffuse and punctuate staining throughout the cytoplasm of approximately half of ileal epithelial cells and nearly all colonic epithelial cells (Figure 3). Examination at higher magnification showed that a
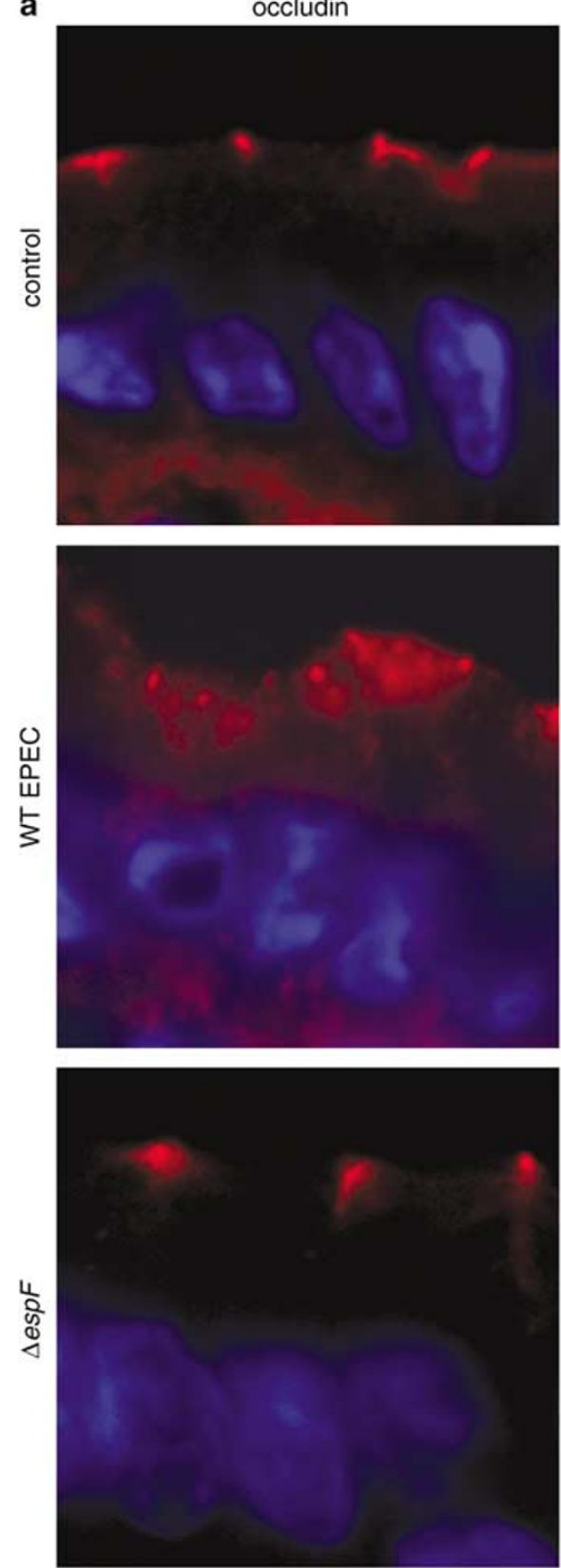

f-actin
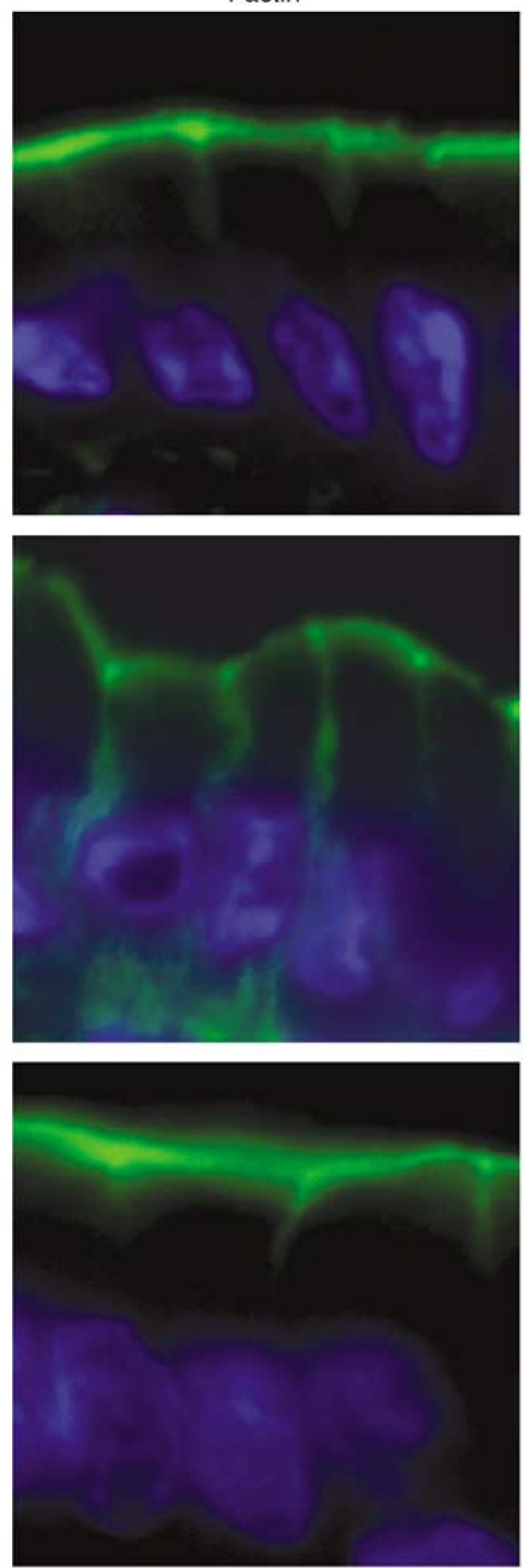

merge
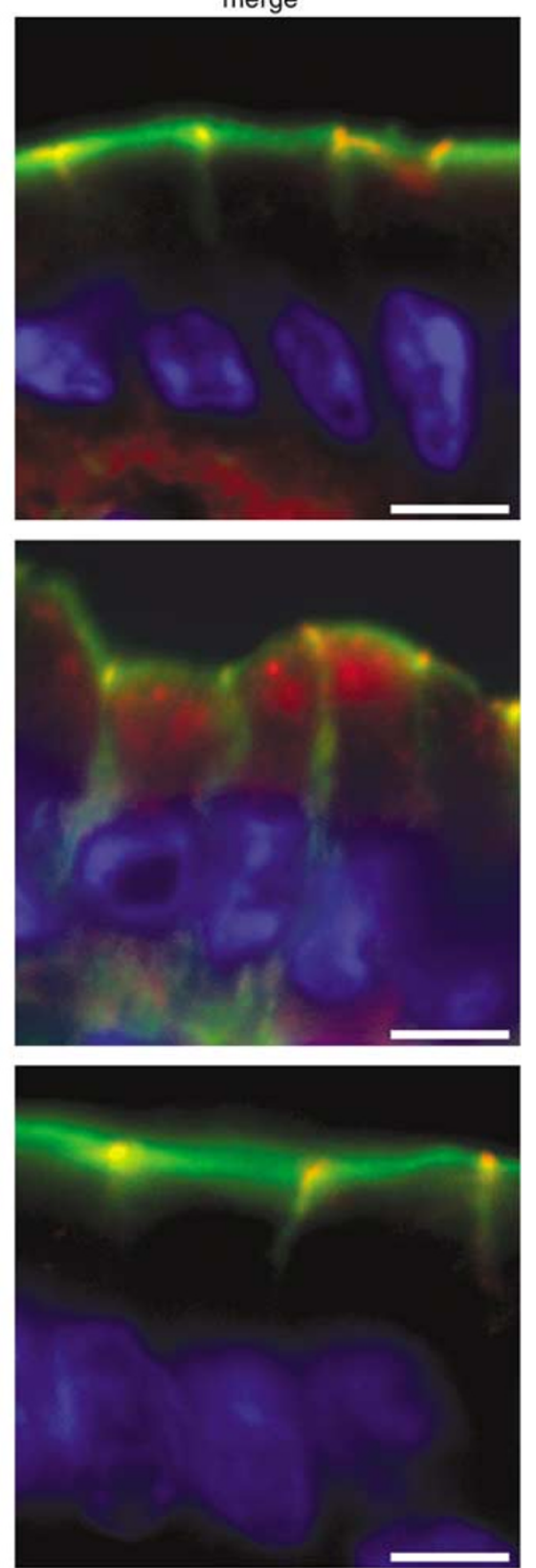

Figure 4 Occludin is present at the TJ and in cytoplasmic pools 1 day after infection with WT EPEC, but not $\Delta e s p F$. Tissues were stained for occludin (red), f-actin (green), and nuclei (blue) as in Figure 3. Higher magnification examination demonstrates that the cytoplasmic pool of occludin that appears after WT EPEC infection is composed of multiple punctuate red dots, consistent with membrane-bound vesicles, in both ileal (a) and colonic (b) epithelium. The distribution of occludin in tissues from control uninfected mice and $\Delta e s p F$ infected mice were similar. Scale bar $=5 \mu \mathrm{m}$. 

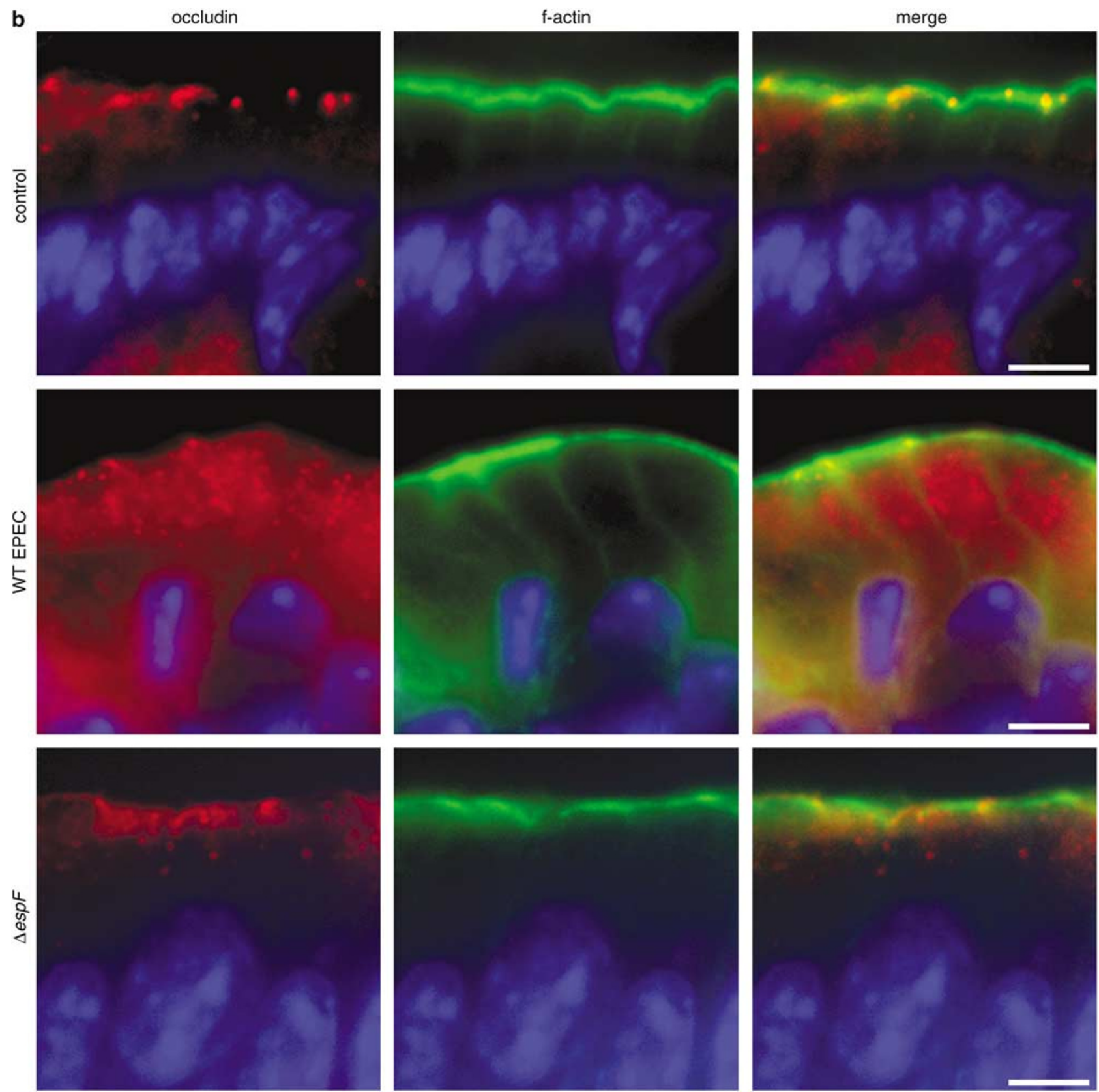

Figure 4 Continued.

both diffuse and punctuate cytoplasmic staining represented foci of intracellular occludin consistent with membrane-bound vesicles (Figure 4). In mice infected with $\Delta e s p F$ for 1 day, occludin was localized only to the $\mathrm{TJ}$ in ileal epithelium (Figures 3 and 4). This distribution was indistinguishable from that of control uninfected mice. In the colon of $\Delta e s p F$-infected mice, occludin was similarly restricted to the TJ in nearly all epithelial cells, but focal alterations in occludin distribution could be identified in rare epithelial cells (Figure 3, arrows).

To determine if alterations in occludin distribution were accompanied by changes in total cellular occludin content we performed Western blots on ileal and colonic tissues from three individual mice per treatment group. These revealed similar occludin protein content across all treatment groups. Although identical total protein loads were applied to the gels, $\beta$-actin content was used to further normalize comparisons. Densitometric analysis of occludin signal normalized to $\beta$-actin signal revealed ratios of $0.46 \pm 0.05,0.41 \pm 0.02$, and $0.48 \pm$ 0.04 for ileal tissue from control, WT EPEC-, and $\Delta e s p F$-infected animals, respectively. Similarly, occludin: $\beta$-actin ratios were not altered in colonic tissues $(0.51 \pm 0.07,0.49 \pm 0.07,0.52 \pm 0.06$, for 
control, WT EPEC-, and $\Delta e s p F$-infected animals, respectively). Thus, the observed occludin redistribution following a 1 day infection with WT EPEC was not associated with altered occludin expression.

Like occludin, the TJ plaque protein ZO-1 was localized with the perijunctional actomyosin ring in control uninfected mice and $\Delta e s p F$-infected mice (Figure 5). However, in contrast to occludin, ZO-1 remained localized to the TJ in WT EPEC-infected mice. Thus, although occludin is redistributed away from the TJ following WT EPEC infection, this does not represent global TJ disruption, as ZO-1 remains precisely localized to the TJ.

\section{Both WT EPEC and $\triangle e s p F$ Disrupt Intestinal Epithelial Barrier Function at 5 Days Postinfection}

The lack of effects of $\Delta e s p F$ on barrier function in vitro can be overcome in part by increasing the multiplicity of infection or the time postinfection. ${ }^{11}$ In order to assess whether the different effects of WT
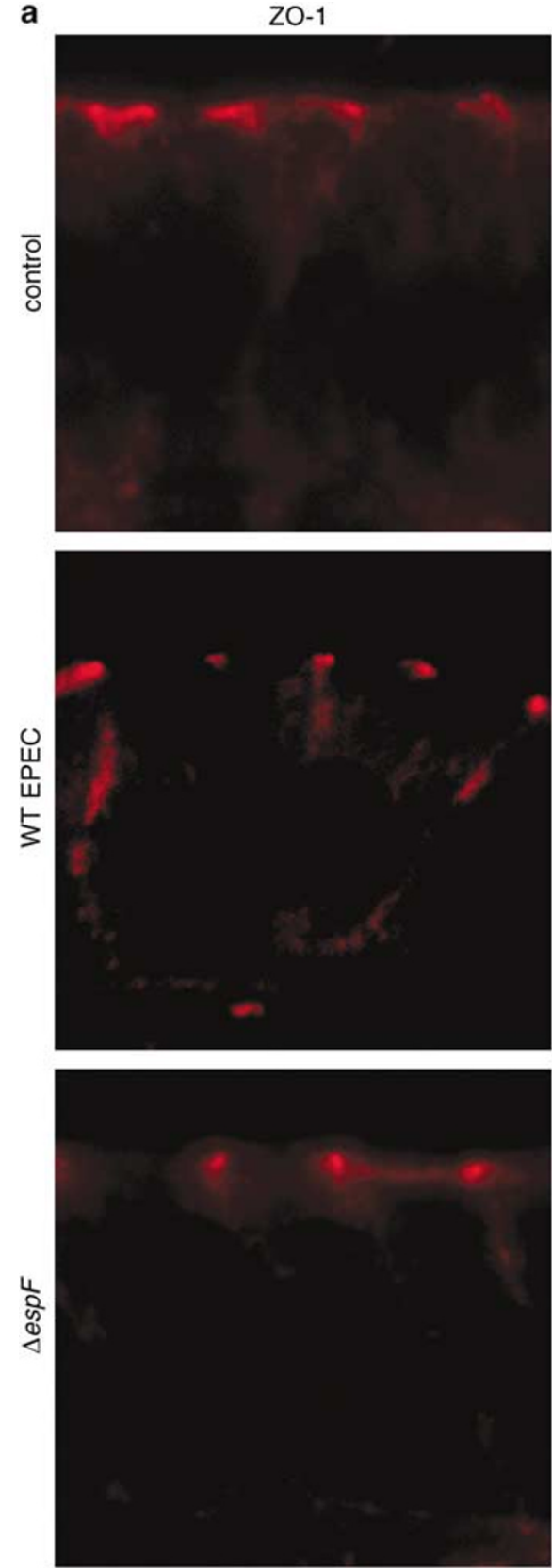

f-actin
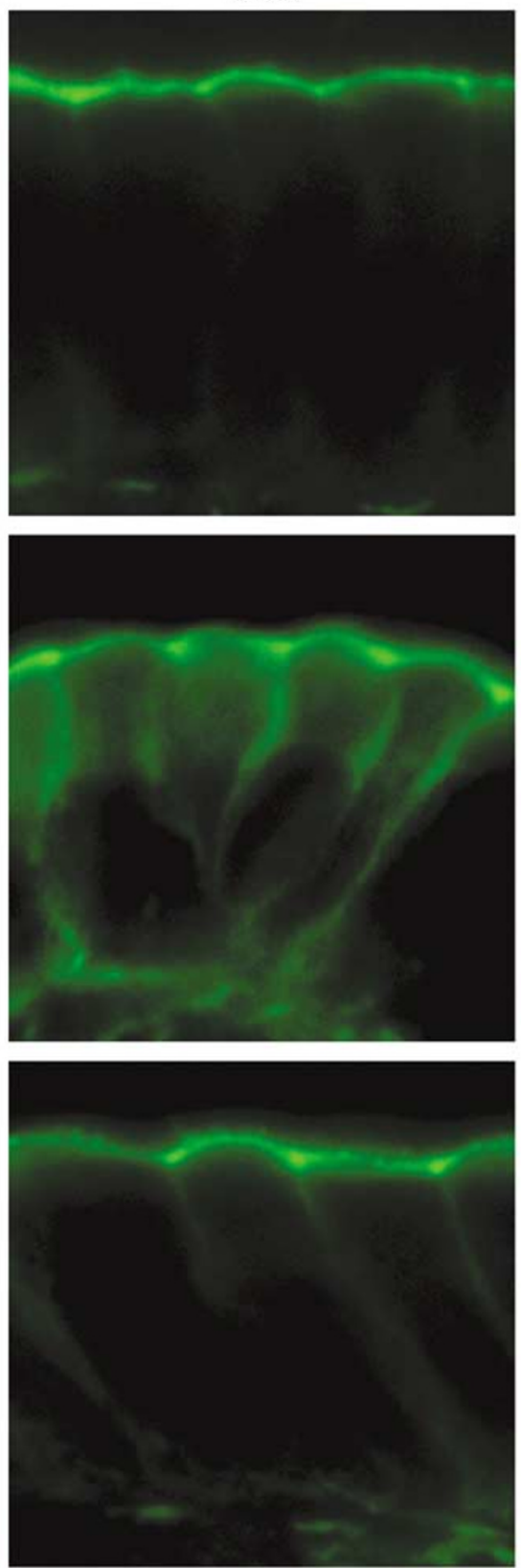

merge
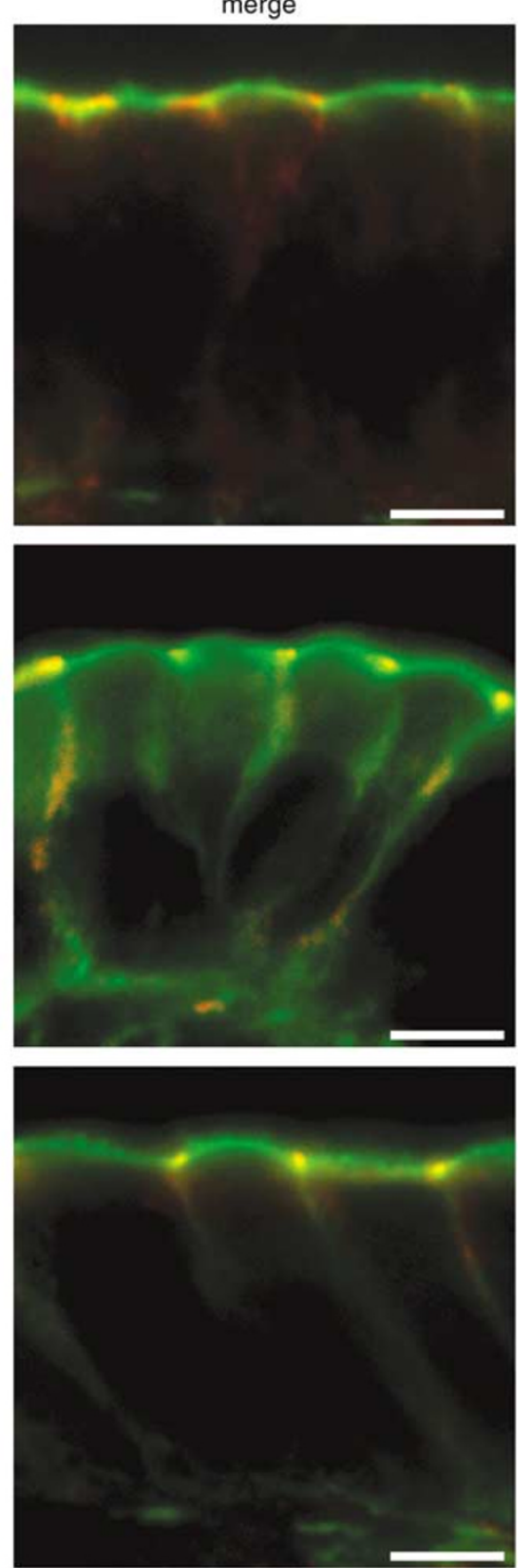

Figure 5 EPEC infection does not alter the distribution of intestinal epithelial ZO-1 after 1 day of infection. Sections of ileum (a) and colon (b) were labeled for ZO-1 (red), f-actin (green), and nuclei (blue). In both ileal and colonic epithelium, ZO-1 was localized to the TJ. In contrast to occludin, this distribution was not altered in tissue from WT EPEC and $\Delta e s p F$-infected mice. Scale bar $=5 \mu \mathrm{m}$. 

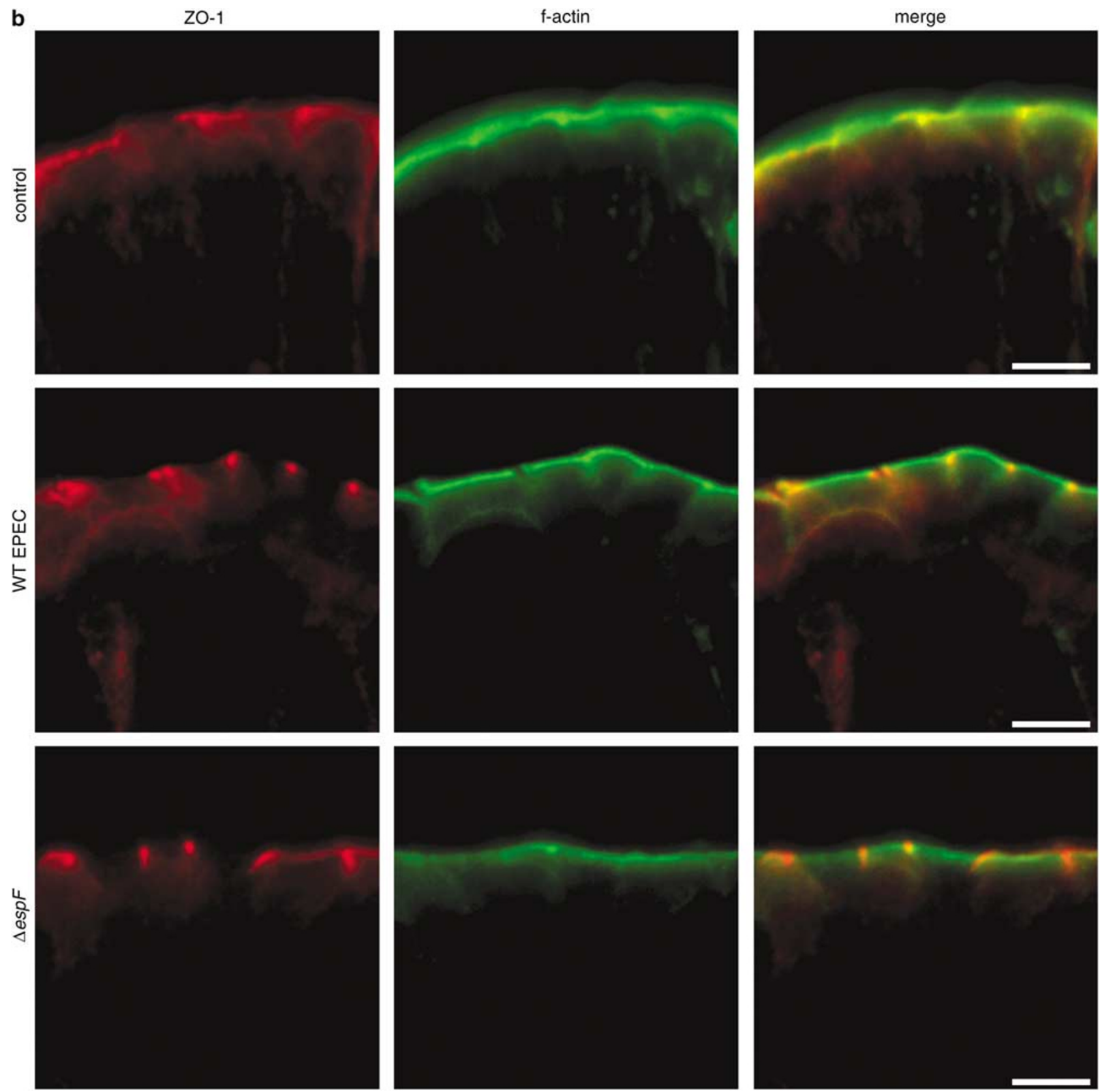

Figure 5 Continued.

EPEC and $\triangle e s p F$ on TJ structure and function seen at 1 day postinfection persisted, Üssing chamber studies were performed at 5 days postinfection. The effect of WT EPEC on intestinal barrier function at 5 days postinfection was similar to that observed after a 1 day infection. Specifically, both the ileum (Figure 6a) and the colon (Figure 6b) of WT EPECinfected mice had a significantly decreased barrier function as compared to uninfected mice. Similar to 1 day postinfection, there were no alterations in $I_{\mathrm{sc}}$ of the ileum or the colon (Figure 7) after 5 days. However, while the barrier function of $\Delta e s p F$ infected tissues was unchanged at 1 day postin- fection, barrier function of the ileum (Figure 6a) and the colon (Figure 6b) was reduced to the same magnitude as that caused by WT EPEC at 5 days.

\section{Epithelial Occludin is Redistributed Following a 5 Day Infection with WT EPEC or $\triangle$ espF}

Our studies suggested that, unlike early (1 day) infections, longer infection (5 days) with $\Delta e s p F$ results in disruption of intestinal barrier function. To determine if the decreased TER was associated with occludin redistribution, as occurred after a 

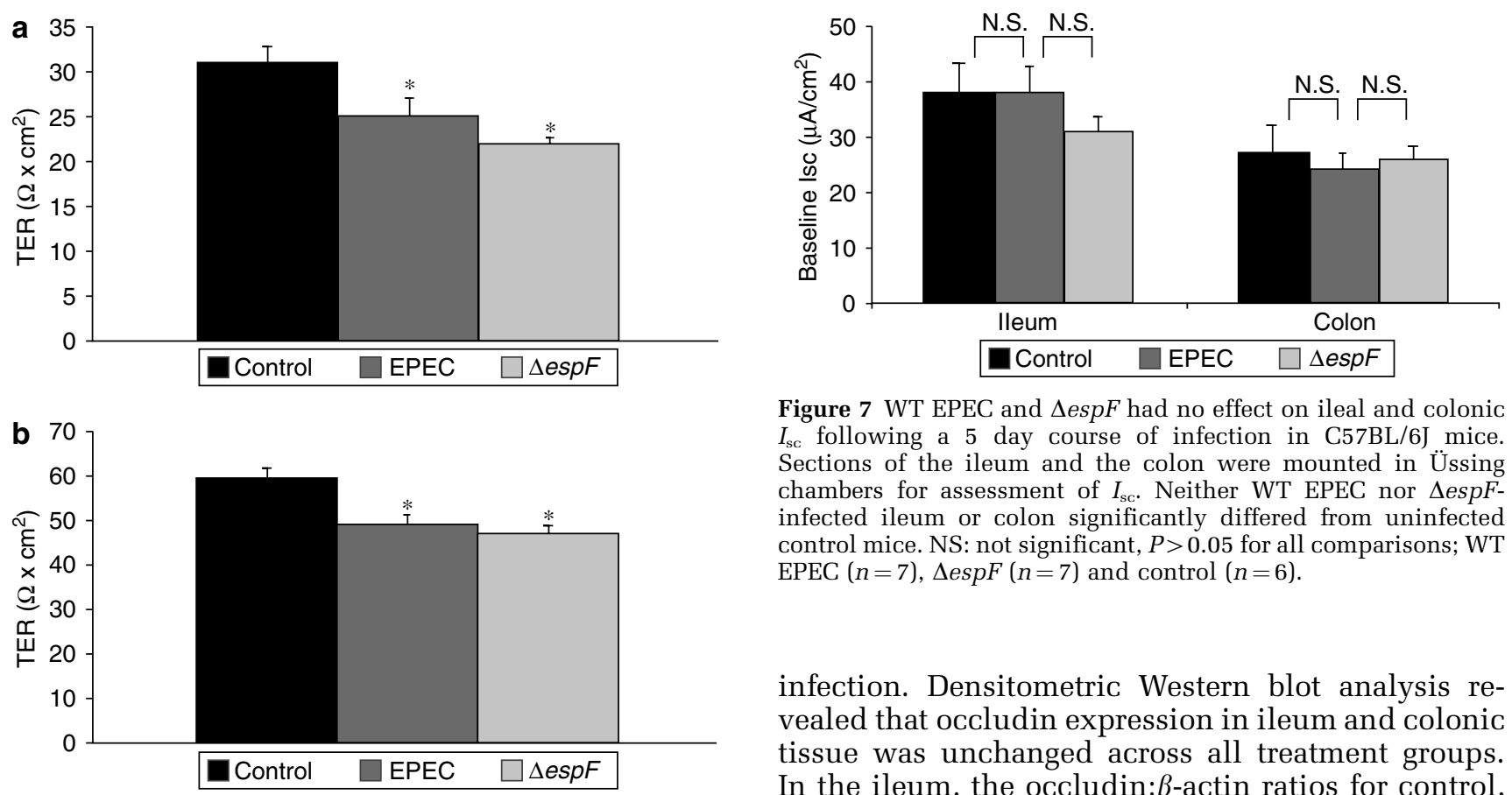

Figure 7 WT EPEC and $\triangle e s p F$ had no effect on ileal and colonic $I_{\text {sc }}$ following a 5 day course of infection in C57BL/6J mice. Sections of the ileum and the colon were mounted in Üssing chambers for assessment of $I_{\mathrm{sc}}$. Neither WT EPEC nor $\Delta e s p F$ infected ileum or colon significantly differed from uninfected control mice. NS: not significant, $P>0.05$ for all comparisons; WT EPEC $(n=7), \Delta \operatorname{espF}(n=7)$ and control $(n=6)$.

Figure 6 Both WT EPEC and $\triangle e s p F$ infection reduces intestinal barrier function at 5 days postinfection. In contrast to the lack of effect of $\triangle e s p F$ on TER after 1 day of infection (Figure 1), both EPEC strains reduced TER by 5 days. (a) Sections of ileal tissue were mounted in Üssing chambers for assessment of TER. Both WT EPEC and $\triangle e s p F$ induced a significant decrease in TER when compared to control uninfected mice. (b) WT EPEC and $\Delta e s p F$ also induced a significant decrease in TER of the colon when compared to control uninfected mice. ${ }^{*} P<0.05$, when compared to control. WT EPEC $(n=7), \Delta \operatorname{espF}(n=7)$ and control $(n=6)$.

1 day infection with WT EPEC, both the ileum and the colon of control mice and WT EPEC or $\triangle e s p F$ infected mice were examined immunohistochemically at day 5 (Figures 8 and 9). Control, uninfected mice again revealed occludin colocalization with the apical perijunctional actomyosin rings in both ileal (Figure 8a) and colonic (Figure 8b) epithelia. In mice infected with WT EPEC, the redistribution of occludin in ileal (Figure 8a) and colonic (Figure 8b) epithelium at 5 days postinfection was similar to that seen at 1 day postinfection. Specifically, there were numerous intracellular occludin-containing vesicular structures. Similar to 1 day infections, there was no significant ZO-1 redistribution following a 5 day infection with WT EPEC (Figure 9). Remarkably, the distributions of occludin and ZO-1 in ileal and colonic epithelium of mice infected with $\Delta e s p F$ for 5 days (Figures 8 and 9) were indistinguishable from the corresponding distributions in mice infected with WT EPEC for 5 days. Thus, occludin distribution in mice infected with $\Delta e s p F$ for 1 or 5 days correlates with preserved or disrupted intestinal barrier function, respectively.

We also assessed total occludin content by Western blots to determine if total occludin expression was altered during this longer 5 day course of

infection. Densitometric Western blot analysis revealed that occludin expression in ileum and colonic tissue was unchanged across all treatment groups. In the ileum, the occludin: $\beta$-actin ratios for control, WT EPEC, and $\Delta e s p F$ were $0.46 \pm 0.02,0.43 \pm 0.04$, $0.43 \pm 0.06$, respectively. Colonic occludin: $\beta$-actin ratios were $0.57 \pm 0.10,0.57 \pm 0.06,0.54 \pm 0.10$ for control, WT EPEC, and $\Delta e s p F$, respectively. In contrast to the changes in occludin localization, EPEC had no effect on the distribution of ZO-1. Since ZO-1 immunofluorescence was unchanged from control in the ileum and the colon at 1 and 5 days postinfection, we elected to assess the amount of ZO-1 expression in a representative group, colonic tissue after 5 days of infection. Similar to occludin expression, there was no alteration in the ratio of ZO-1: $\beta$-actin in the colon at 5 days postinfection $(0.41 \pm 0.08,0.46 \pm 0.10,0.40 \pm 0.06$ for control, WT EPEC, and $\Delta e s p F$, respectively) Thus, 5 day EPEC infections do not induce changes in total occludin or ZO-1 content within the intestinal epithelium.

\section{WT and $\Delta e s p F$ Increase Cytokine Expression at 5 Days Postinfection}

The observed disruption of intestinal barrier function and structural alteration of the TJ following 5 days of infection with both WT EPEC and $\Delta e s p F$ led us to examine the potential for nonbacterial factors to regulate intestinal barrier function. Initially, ileal and colonic tissues from control, WT EPEC, and $\Delta e s p F$-infected mice were examined histologically following 5 days of infection. Ileal (Figure 10a) and colonic mucosa demonstrated crypt cell expansion at 5 days, but not 1 day, after infection with WT EPEC and $\triangle e s p F$. We therefore assessed expression of the proinflammatory cytokine TNF- $\alpha$ in ileal and colonic tissue at 1 or 5 days postinfection. At 1 day after infection with either WT EPEC or $\Delta e s p F$ there was no difference in TNF- $\alpha$ mRNA expression in 

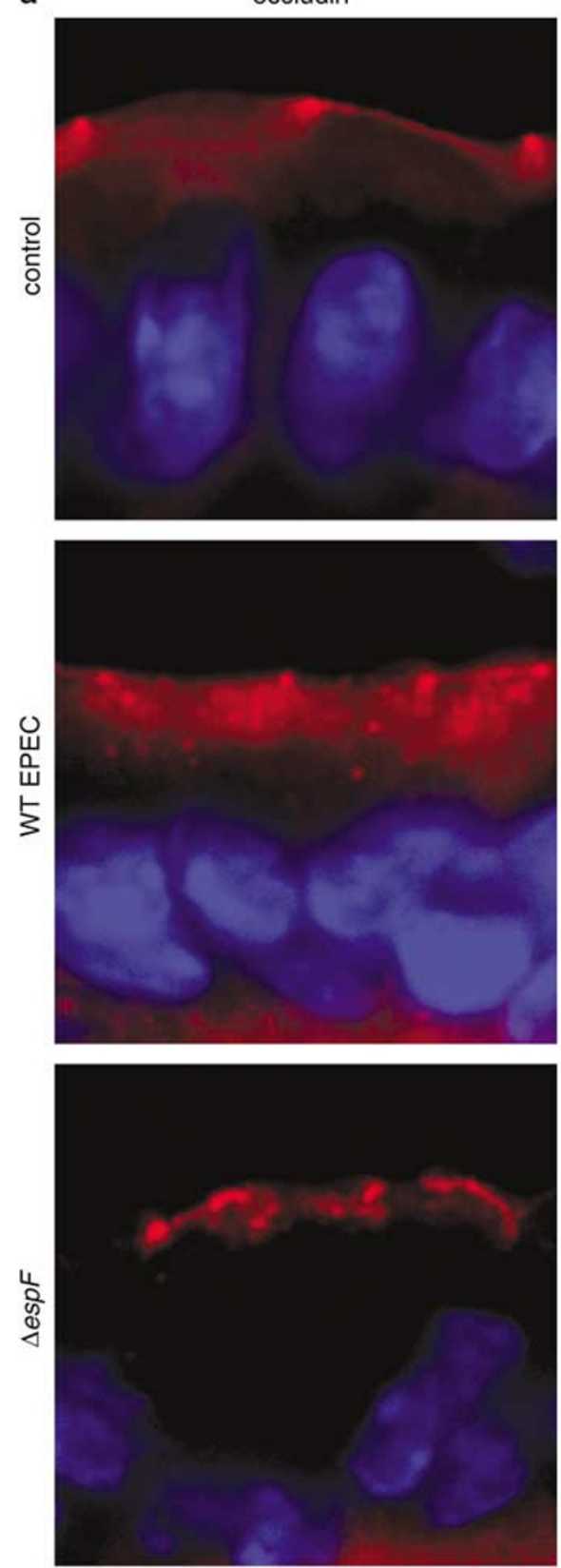

f-actin
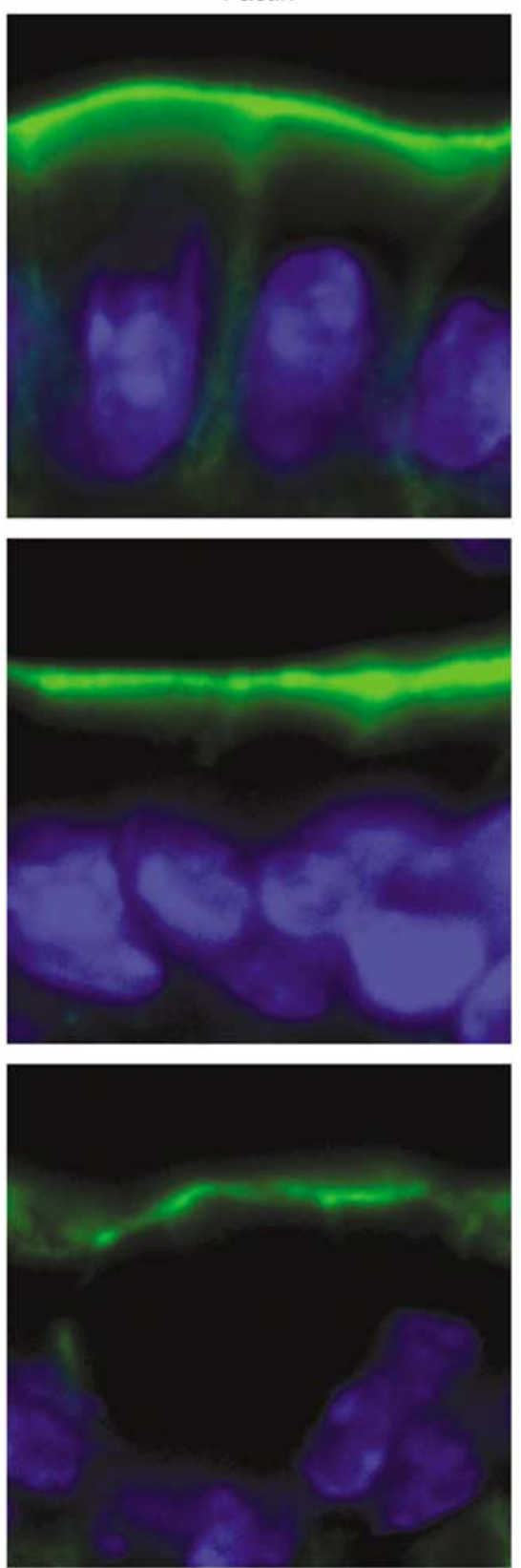

merge
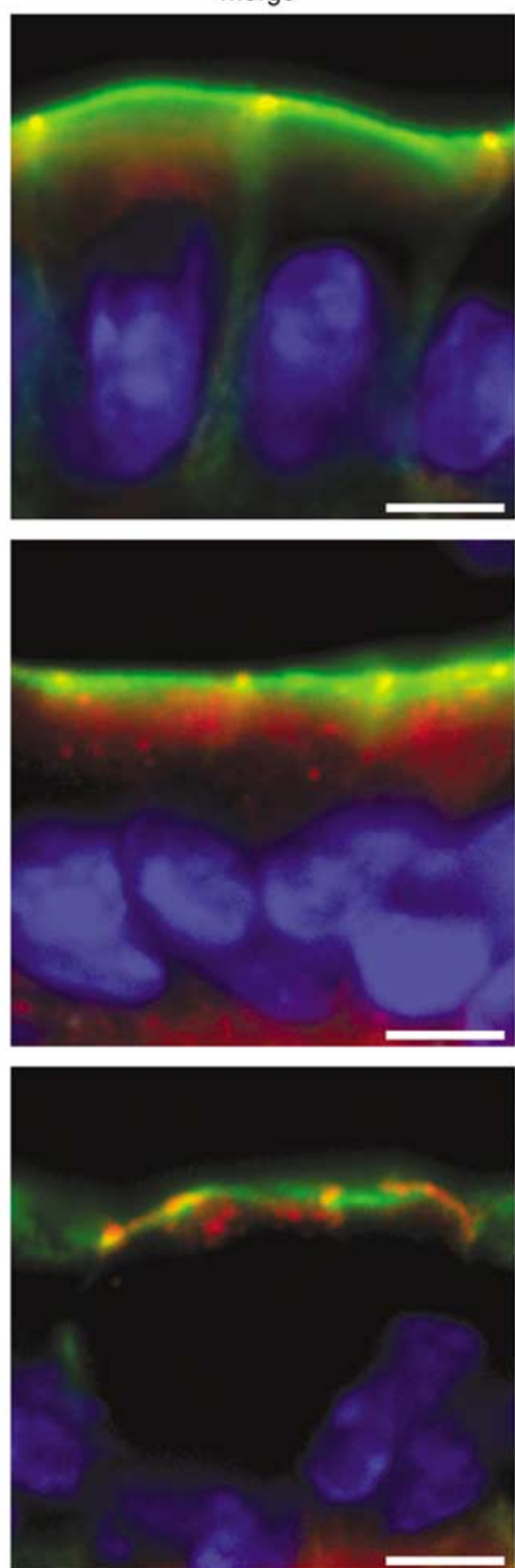

Figure 8 After prolonged infection (5 days), intestinal epithelial occludin is redistributed to a cytoplasmic pool in both WT EPEC and $\Delta e s p F$-infected mice. Ileum (a) and colon (b) from control uninfected mice and mice infected with WT EPEC or $\Delta e s p F$ for 5 days were stained for occludin (red), f-actin (green), and nuclei (blue). Like 1 day of infection, 5 days of infection with WT EPEC caused the appearance of a cytoplasmic pool of occludin in ileal and colonic epithelium. However, in contrast to the results after 1 day of infection, 5 days of infection with $\triangle e s p F$ also induced occludin redistribution. This occurred in both ileal and colonic epithelium and was similar to that occurring in WT EPEC-infected mice. Scale bar $=5 \mu \mathrm{m}$.

ileal or colonic tissue from any treatment group (Figure 10b). In contrast, at 5 days postinfection, both WT EPEC and $\Delta e s p F$ increased TNF- $\alpha$ mRNA expression in the ileum and the colon (Figure 10b). Thus, there is significant immune activation in both the ileum and the colon at 5 days postinfection, but not at 1 day postinfection with WT EPEC or $\Delta e s p F$. This nonbacterial factor has the potential to regulate intestinal barrier function and TJ structure..$^{16,27,28}$

\section{Discussion}

The pathophysiology of EPEC-induced diarrhea is poorly understood. Our current knowledge of the mechanisms by which this noninvasive pathogen causes diarrhea is largely based on studies from cultured intestinal epithelial cell monolayers infected with WT EPEC or specific mutant strains. These models have demonstrated that EPEC de- 

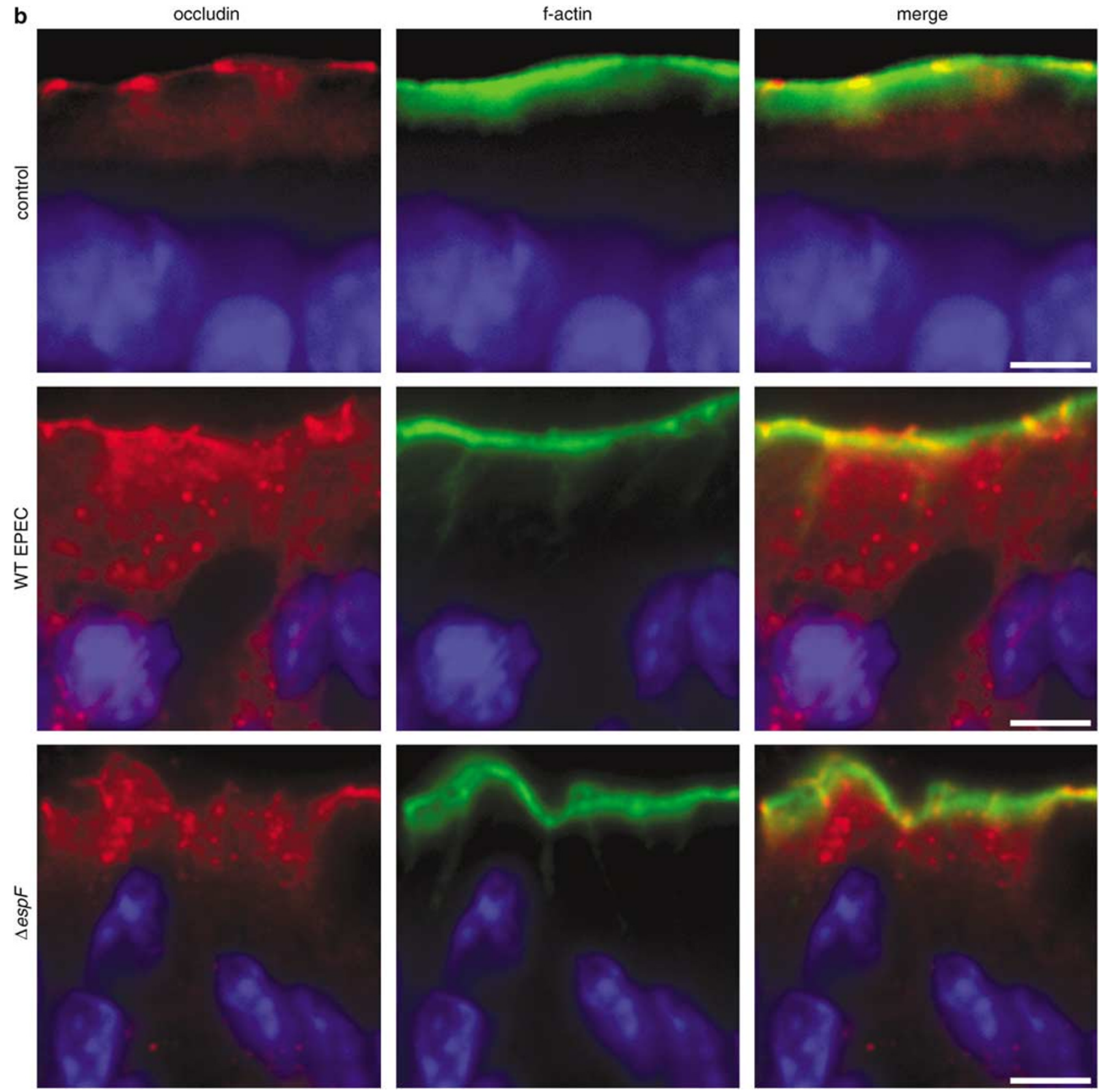

Figure 8 Continued.

creases intestinal barrier function, disrupts cell polarity, alters ion transport, and stimulates the inflammatory response..$^{71,12,14-17,29,30}$ In vitro, the EPEC-induced barrier dysfunction is attributed in large part to the secreted effector, EspF..11,13,18 However, in vivo analysis of $\mathrm{TJ}$ structure and function and the role of EspF in this process has been delayed due to the lack of a small animal model of EPEC infection. We recently characterized the C57BL/6J mouse as a model of EPEC infection ${ }^{26}$ and utilizing this model we demonstrate here for the first time that EPEC infection in vivo also results in disruption of intestinal barrier function. We also show that these functional measurements correlate with alterations in $\mathrm{TJ}$ architecture, specifically redistribution of occludin. Furthermore, these changes in intestinal barrier function and TJ structure are mediated by EspF at early time points (1 day) after infection, but not at later time points (5 days) of infection. Thus, at early time points (1 day), our in vivo data correlates with our in vitro studies demonstrating that disruption of intestinal epithelial barrier function and the redistribution of $\mathrm{TJ}$ proteins is EspF dependent. In contrast, our data also show that at 5 days postinfection there is a generalized tissue response that includes crypt cell 
expansion and increased TNF- $\alpha$ mRNA expression in both the ileum and colon. Sequelae of this generalized response may contribute to the structural and functional TJ disruption noted at 5 days postinfection.

Unresolved is the mechanism by which EspF perturbs TJ structure and function. A dose-dependent correlation between EspF expression, disruption of the TJ barrier, and redistribution of occludin has been demonstrated previously. ${ }^{11}$ Additionally, EspF is targeted to the host mitochondria and contributes to enhanced cell death in vitro. ${ }^{31,32}$
Conversely, in a rabbit EPEC model of infection, there were no effects on, or even a slight decrease in the level of apoptosis in the rabbit intestine. ${ }^{33} \mathrm{EspF}$ also interacts with the intermediate filament protein CK18 and increases its solubility by enhancing its interaction with 14-3-3, ultimately leading to collapse of the intermediate filament network of intestinal epithelial cells. While this phenotype likely contributes to EPEC pathogenesis, ${ }^{19}$ there is no evidence that intermediate filament disruption alters TJ barrier function. In fact, keratin 8 null mice a
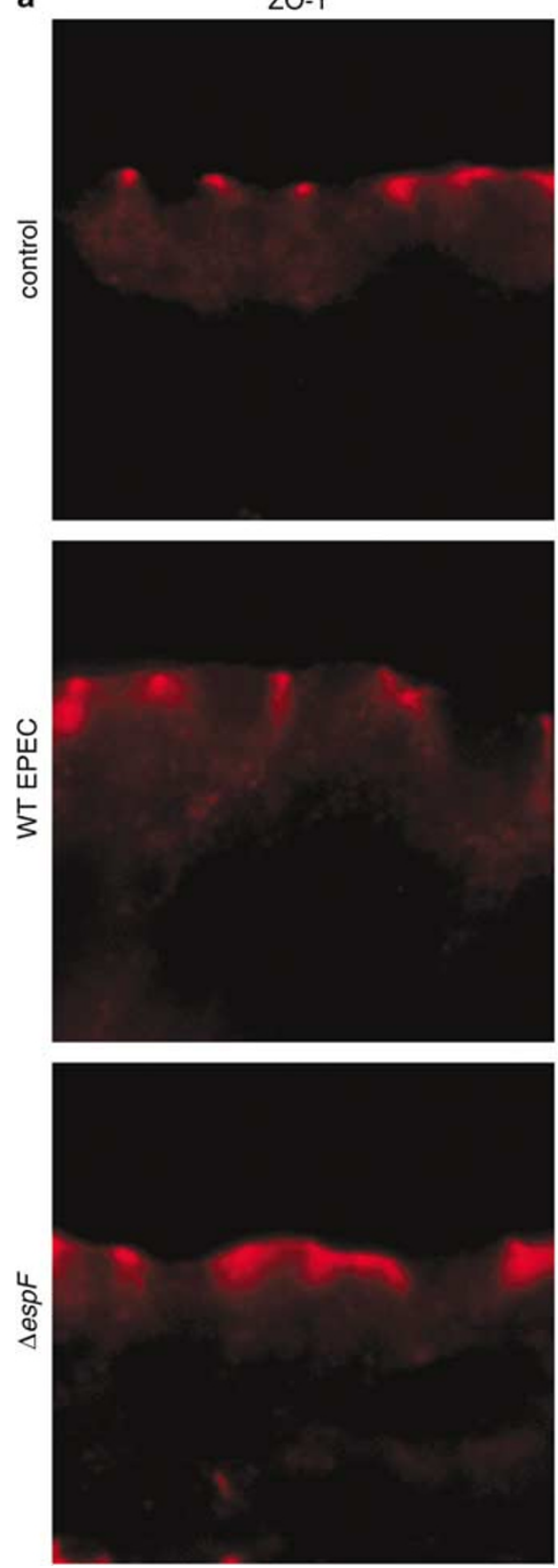

f-actin
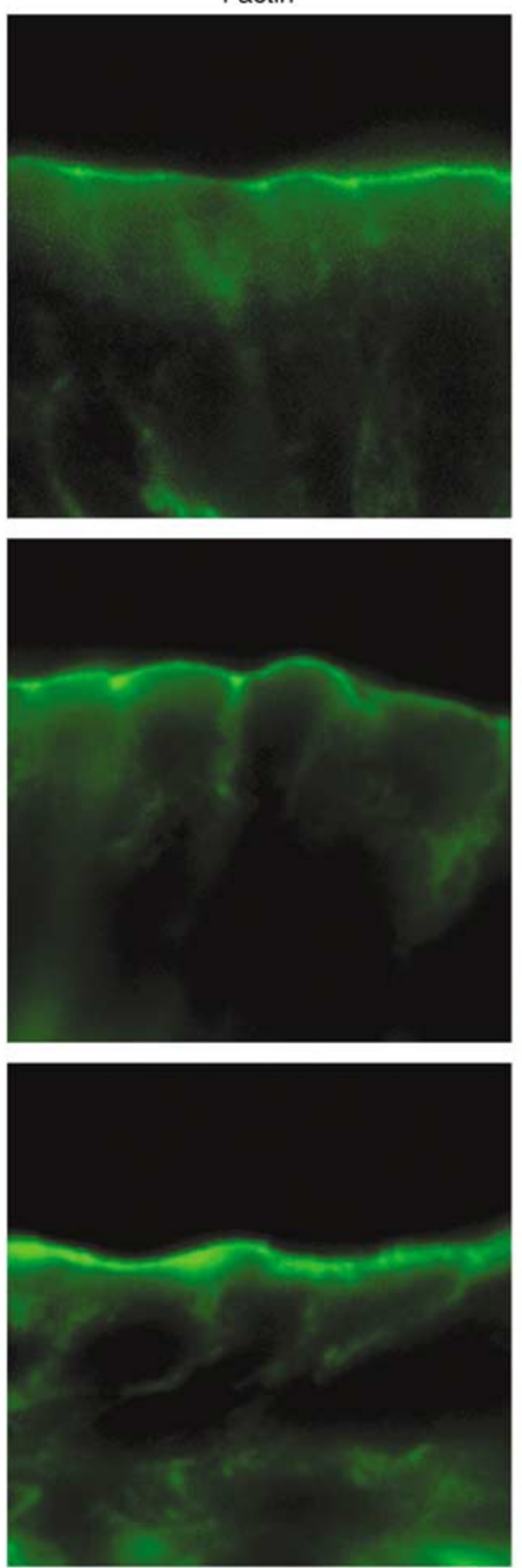

merge
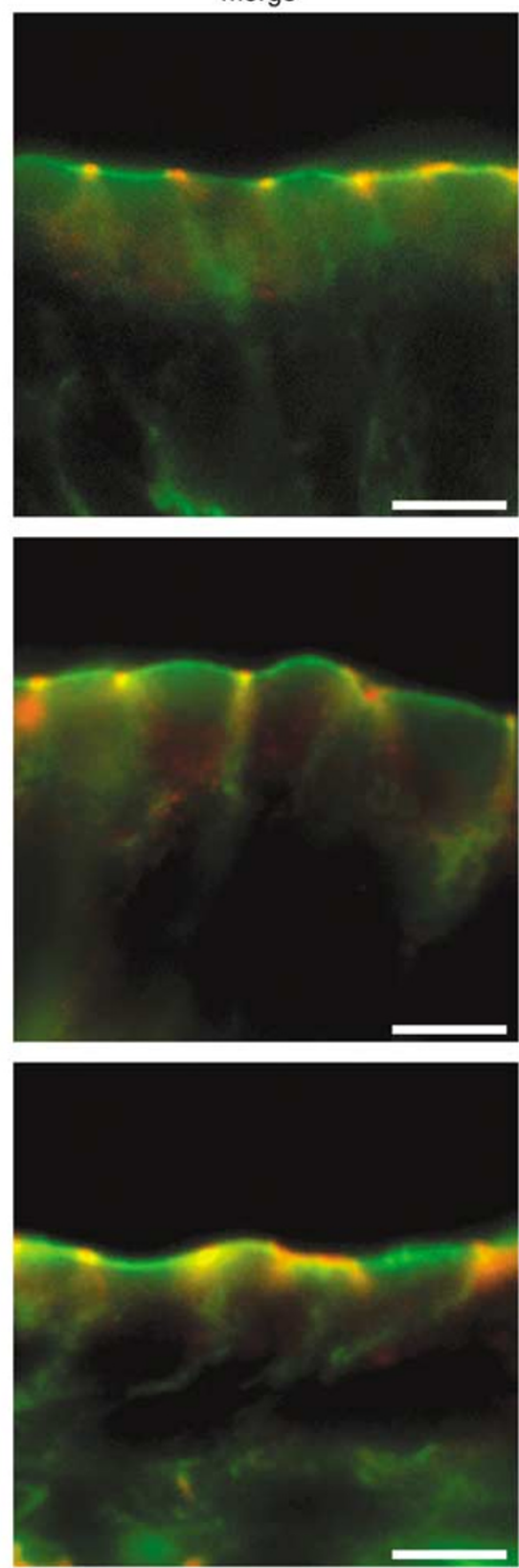

Figure 9 Neither WT EPEC nor $\triangle e s p F$ affect ZO-1 distribution after prolonged infection (5 days). Sections of ileum (a) and colon (b) were labeled for ZO-1 (red), f-actin (green), and nuclei (blue). In both ileal (a) and colonic (b) epithelium, ZO-1 was localized to the TJ. This distribution was not altered in tissue from mice infected with WT EPEC or $\Delta e s p F$ for 5 days. Scale bar $=5 \mu \mathrm{m}$. 
b
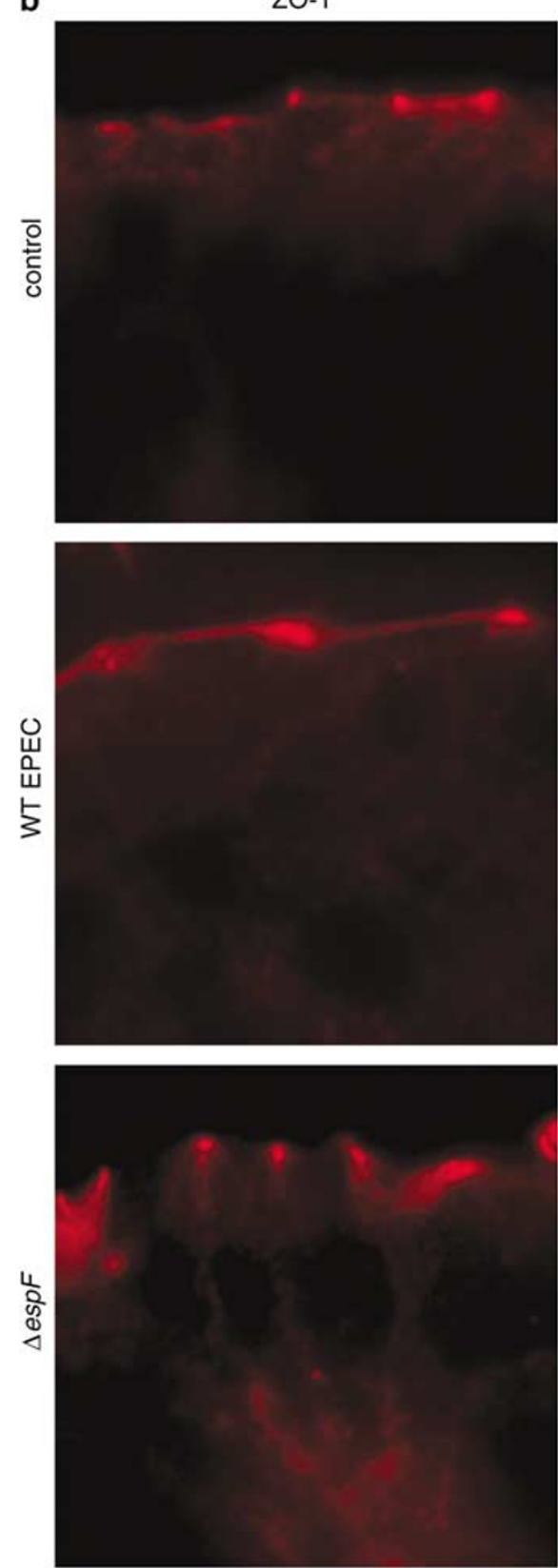

f-actin
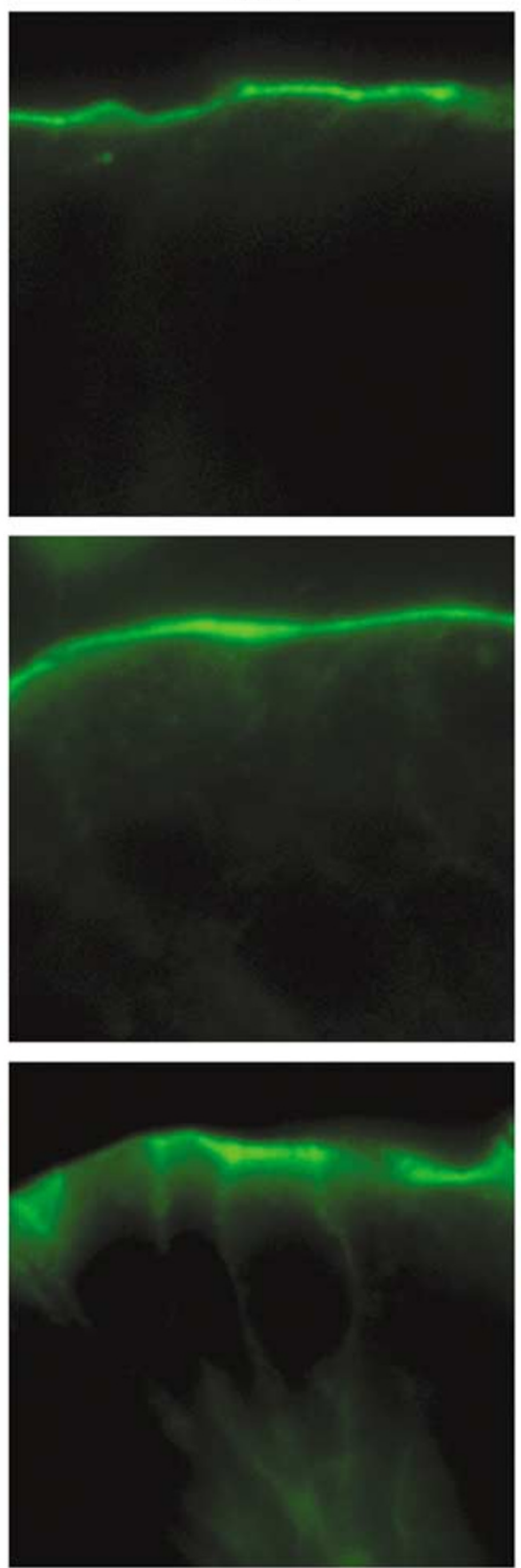

merge
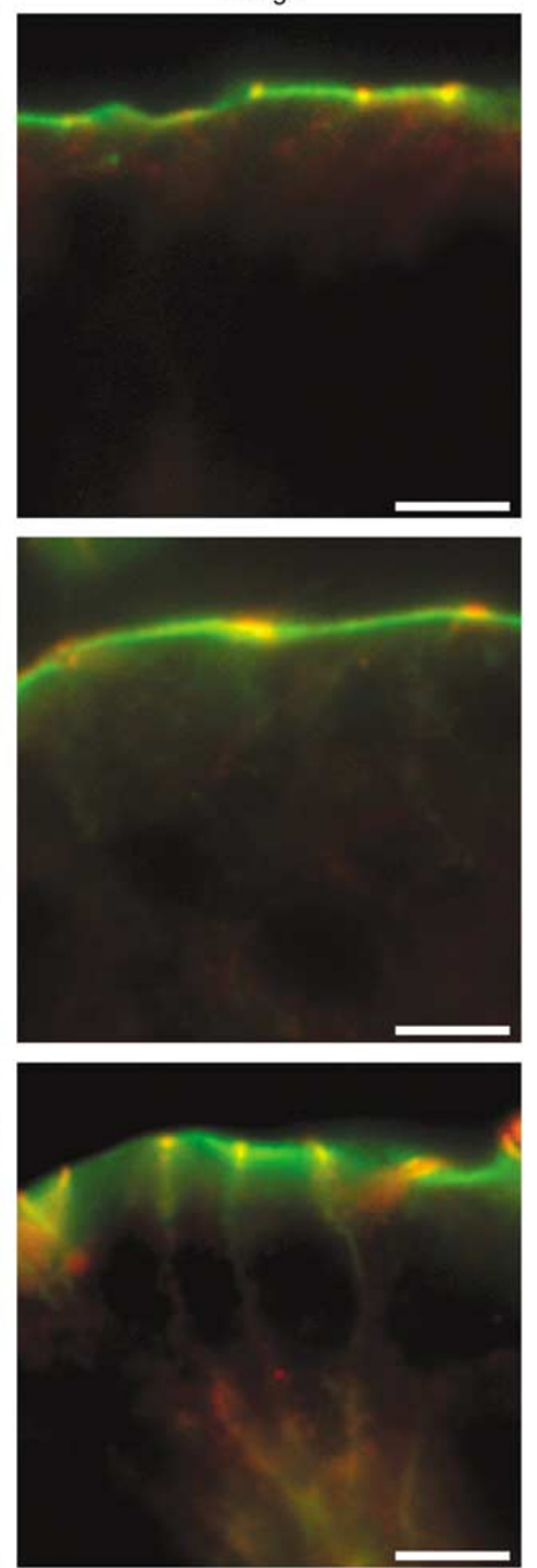

Figure 9 Continued.

that are devoid of enterocytic intermediate filaments have completely normal intestinal barrier function. Instead, intestinal electrolyte transport activities are altered in these mice, likely due to the mistargeting of ion transporters. ${ }^{34}$ Thus, disruption of the intestinal intermediate filament network by EPEC may not be related to the perturbation of TJs but may contribute to the previously reported alterations in electrolyte transport. $^{9}$

In contrast to a 1 day infection, a 5 day course of infection with $\Delta e s p F$ had the same effect as WT EPEC on intestinal barrier function and occludin localization. This finding suggests that different factors may govern these changes at early vs later time points after infection. EspF is clearly important for initiating TJ alterations both in vitro and in vivo. Yet, exact correlation of infection times between in vitro models and in vivo models cannot be achieved. In order for intestinal colonization to be established in vivo, microbial pathogens must overcome factors interfering with adherence including the glycocalyx, mucins, normal flora, and intestinal peristalsis and fluid movement, that are not present in vitro. Therefore, the exact time at which colonization levels achieved in vitro mirror those of the murine intestine cannot be precisely determined. This issue 


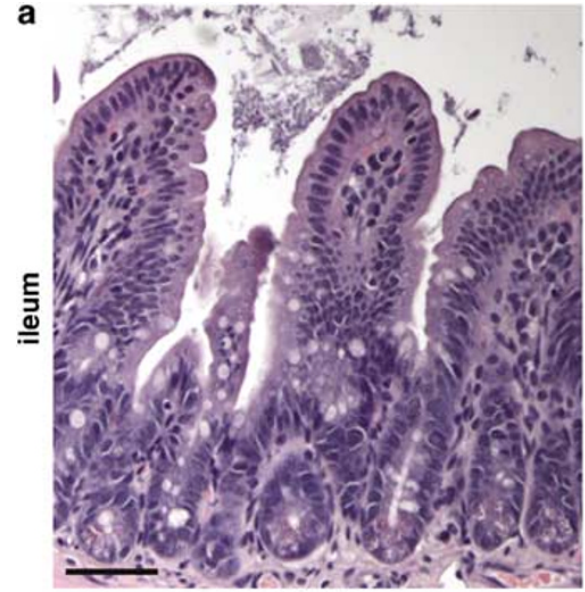

control

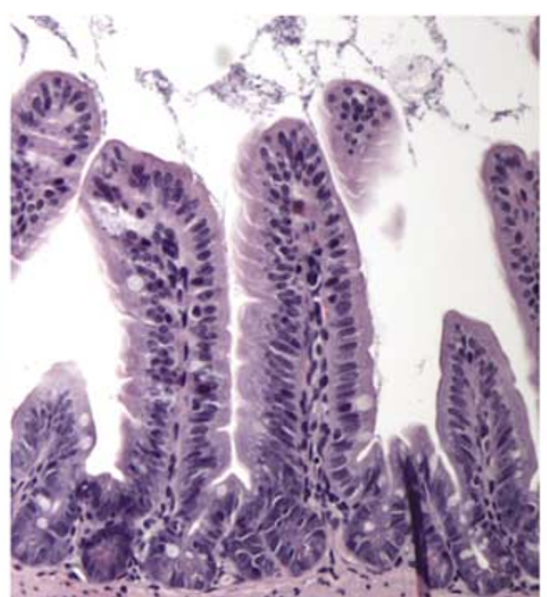

1 day WT EPEC

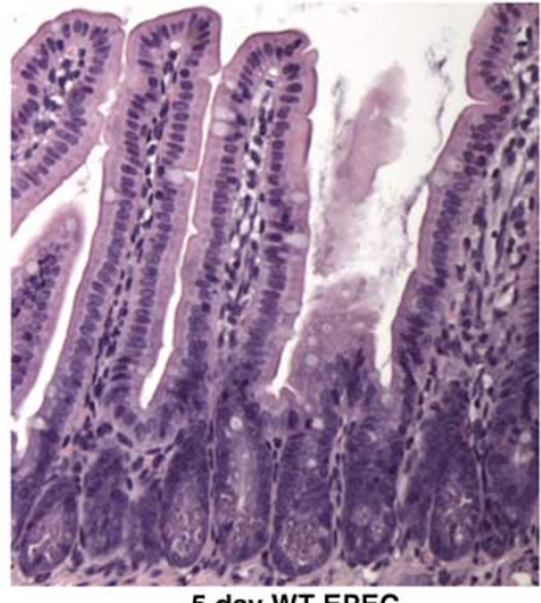

5 day WT EPEC

b

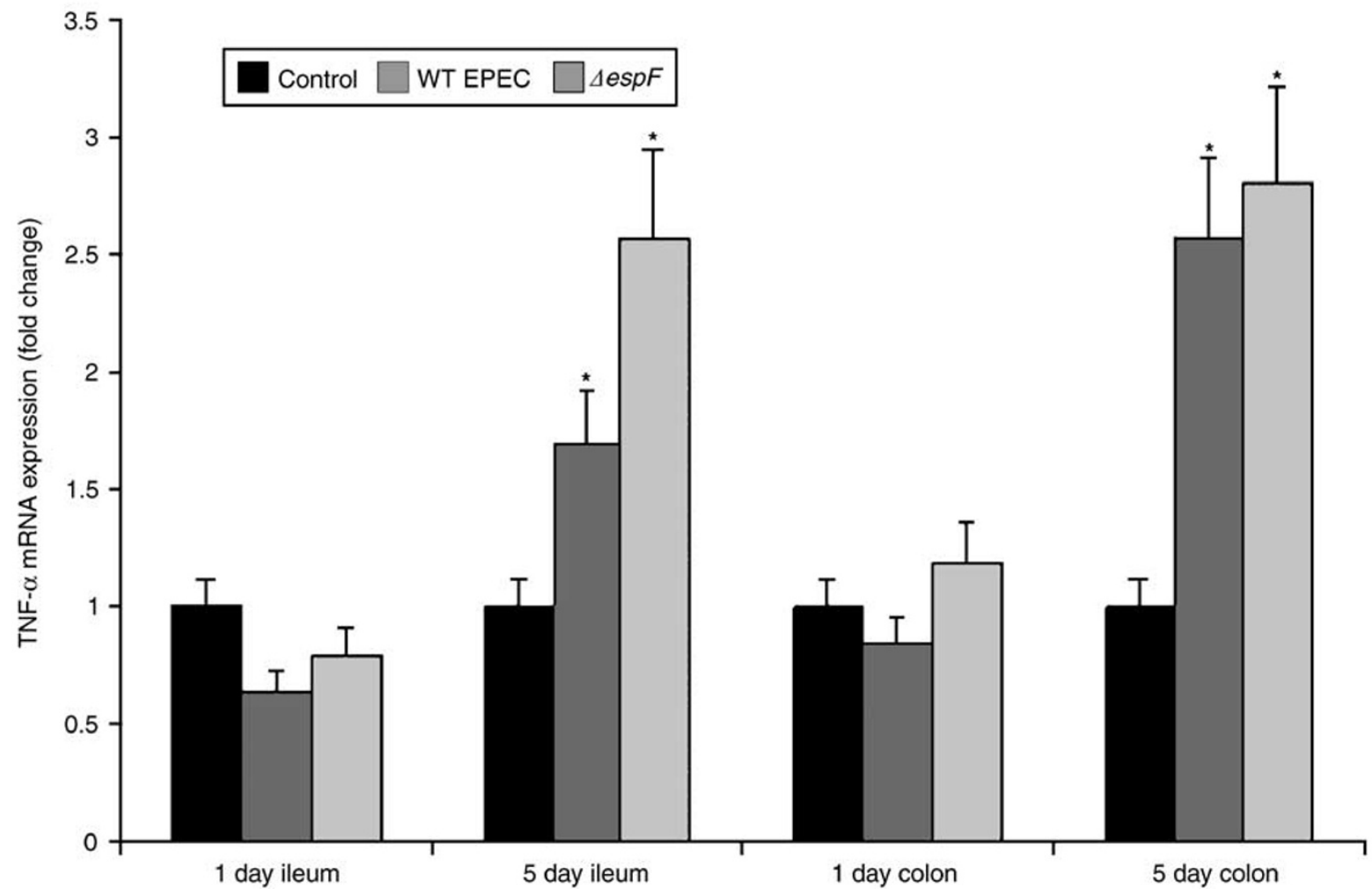

Figure $10 \mathrm{WT}$ EPEC and $\triangle e s p F$ induce a generalized tissue response 5 days postinfection. (a) H\&E staining was performed on sections of ileal mucosa following a 5 day infection with WT EPEC. Ileal tissue from mice infected for 1 day with WT EPEC appeared similar to control (uninfected) ileal mucosa. Conversely, following a 5 day course of infection with WT EPEC, there was crypt cell expansion. (b) Real-time RT-PCR analysis of TNF- $\alpha$ mRNA expression at 1 day postinfection showed that both ileum and colon had similar levels of TNF- $\alpha$ mRNA expression in all three treatment groups. After 5 days, both WT EPEC and $\Delta e s p F$ infection resulted in significant increases in TNF- $\alpha$ mRNA expression in ileum and colon. ${ }^{*} P<0.05$, when compared to control and when compared to corresponding 1 day infection treatment.

is potentially important since the lack of effects of $\Delta e s p F$ on TJs in vitro can be overcome to some degree by increasing the multiplicity of infection (MOI). ${ }^{11}$ Interestingly, the level of colonization in vivo was not increased at day 5 when compared to day 1 postinfection (Table 1). Thus, this indicates that increased MOI is not responsible for the effects of $\triangle e s p F$ at 5 days postinfection.
It is plausible however, that by 5 days postinfection, nonbacterial factors contribute to TJ disruption. We have previously reported that EPEC infection triggers an inflammatory response both in vitro $^{29,35}$ and in vivo. ${ }^{26}$ In vivo, this is evidenced by an increase in the number of neutrophils, intraepithelial leukocytes, and goblet cells in the colon following a 10 day course of infection with WT 
EPEC. ${ }^{26}$ Here we demonstrate that there is crypt cell expansion and an increase in TNF- $\alpha$ expression in the ileum and the colon following a 5 day infection with WT EPEC or $\Delta e s p F$. This is an important finding as the deleterious effects of inflammatory mediators, particularly TNF- $\alpha$ and interferon gamma (IFN- $\gamma$ ), on intestinal barrier function and TJ structure have been previously documented in vitro. ${ }^{16,27,28,36-42}$ In fact, enterocytes from rabbits infected with rabbit EPEC exhibit an increase in mRNA expression of TNF- $\alpha$, IL-1 $\beta$, and IL-8 following a six day infection. ${ }^{43}$ Thus, the potential for inflammatory mediators to perturb TJ intestinal barrier function during infection with WT EPEC or mutant EPEC strains cannot be ignored. Additionally, the transepithelial migration of neutrophils through TJs perturbs intestinal epithelial barrier function. ${ }^{44-48}$ The factors governing EPEC-induced inflammation are not fully defined. It has been reported that EPEC flagellin induces IL-8 release in T84-cell monolayers. ${ }^{49}$ Also, a heightened inflammatory response in infant rabbits infected with EHEC $\Delta e s p F$ for 7 days was recently described.$^{50}$ It is obvious that additional studies concerning the role of EPEC-effector proteins in regulating inflammation are needed.

The disruption of TJ barrier function by EPEC in vivo has important clinical implications. Under normal circumstances, water is passively absorbed following the absorption of solutes. Alteration of the TJ disrupts electrolyte processes such as the sodium gradient. ${ }^{51}$ Disruption of the sodium gradient allows sodium concentrations to equilibrate between the serosal surface and the luminal surface. The resultant increase in luminal sodium above normal physiological concentrations allows the accumulation of water in the intestinal lumen and enhances diarrhea. Thus, EPEC-induced alterations of the TJ may be clinically relevant.

In conclusion, we demonstrate here for the first time that EPEC disrupts TJ barrier function and structure in vivo. Furthermore, we show that these changes are $\mathrm{EspF}$ dependent at early time points after infection (1 day), but that at later times ( 5 days), other factors, potentially both pathogen- and host derived, may contribute to disruptions in intestinal barrier function. The availability of a small animal model to study the pathophysiology of EPEC infection should promote our understanding of this important human organism. Insights into the mechanisms responsible for EPEC-induced alteration of intestinal barrier function as well as the factors that govern intestinal inflammation can be potentially gained from the use of this murine model of EPEC infection.

\section{Acknowledgements}

This work was presented in preliminary form at the annual meeting of the American Gastroenterological
Association in May 2005. We thank Dr Suzana Savkovic for her technical guidance during initiation of this project. This work was supported by grants from the National Institutes of Health \#DK50694 and \#DK58964 to GH, DK61931 and DK68271 to JRT, and the Department of Veterans Affairs (Merit award) to GH. DRC is a predoctoral fellow of the National Institutes of Health (The University of Chicago Medical Scientist Training Program T32 GM07281).

\section{References}

1 Knutton S, Lloyd DR, McNeish AS. Adhesion of enteropathogenic Escherichia coli to human intestinal enterocytes and cultured human intestinal mucosa. Infect Immun 1987;55:69-77.

2 Hinson G, Knutton S, Lam-Po-Tang MK, et al. Adherence to human colonocytes of an Escherichia coli strain isolated from severe infantile enteritis: molecular and ultrastructural studies of a fibrillar adhesin. Infect Immun 1987;55:393-402.

3 McDaniel TK, Jarvis KG, Donnenberg MS, et al. A genetic locus of enterocyte effacement conserved among diverse enterobacterial pathogens. Proc Natl Acad Sci USA 1995;92:1664-1668.

4 Jarvis KG, Giron JA, Jerse AE, et al. Enteropathogenic Escherichia coli contains a putative type III secretion system necessary for the export of proteins involved in attaching and effacing lesion formation. Proc Natl Acad Sci USA 1995;92:7996-8000.

5 Kenny B, Finlay BB. Protein secretion by enteropathogenic Escherichia coli is essential for transducing signals to epithelial cells. Proc Natl Acad Sci USA 1995;92:7991-7995.

6 Savkovic SD, Koutsouris A, Hecht G. Attachment of a noninvasive enteric pathogen, enteropathogenic Escherichia coli, to cultured human intestinal epithelial monolayers induces transmigration of neutrophils. Infect Immun 1996;64:4480-4487.

7 Savkovic SD, Koutsouris A, Hecht G. Activation of NF-kappaB in intestinal epithelial cells by enteropathogenic Escherichia coli. Am J Physiol 1997;273: C1160-C1167.

8 Hecht G, Koutsouris A. Enteropathogenic E. coli attenuates secretagogue-induced net intestinal ion transport but not Cl-secretion. Am J Physiol 1999;276: G781-G788.

9 Hecht G, Hodges K, Gill RK, et al. Differential regulation of $\mathrm{Na}+/ \mathrm{H}+$ exchange isoform activities by enteropathogenic E. coli in human intestinal epithelial cells. Am J Physiol Gastrointest Liver Physiol 2004; 287:G370-G378.

10 Hecht G, Marrero JA, Danilkovich A, et al. Pathogenic Escherichia coli increase Cl- secretion from intestinal epithelia by upregulating galanin-1 receptor expression. J Clin Invest 1999;104:253-262.

11 McNamara BP, Koutsouris A, O’Connell CB, et al. Translocated EspF protein from enteropathogenic Escherichia coli disrupts host intestinal barrier function. J Clin Invest 2001;107:621-629.

12 Muza-Moons MM, Schneeberger EE, Hecht GA. Enteropathogenic Escherichia coli infection leads to appearance of aberrant tight junctions strands in the 
lateral membrane of intestinal epithelial cells. Cell Microbiol 2004;6:783-793.

13 Dean P, Kenny B. Intestinal barrier dysfunction by enteropathogenic Escherichia coli is mediated by two effector molecules and a bacterial surface protein. Mol Microbiol 2004;54:665-675.

14 Simonovic I, Arpin M, Koutsouris A, et al. Enteropathogenic Escherichia coli activates ezrin, which participates in disruption of tight junction barrier function. Infect Immun 2001;69:5679-5688.

15 Simonovic I, Rosenberg J, Koutsouris A, et al. Enteropathogenic Escherichia coli dephosphorylates and dissociates occludin from intestinal epithelial tight junctions. Cell Microbiol 2000;2:305-315.

16 Zolotarevsky Y, Hecht G, Koutsouris A, et al. A membrane-permeant peptide that inhibits MLC kinase restores barrier function in in vitro models of intestinal disease. Gastroenterology 2002;123:163-172.

17 Tomson FL, Koutsouris A, Viswanathan VK, et al. Differing roles of protein kinase C-zeta in disruption of tight junction barrier by enteropathogenic and enterohemorrhagic Escherichia coli. Gastroenterology 2004; 127:859-869.

18 Elliott SJ, O’Connell CB, Koutsouris A, et al. A gene from the locus of enterocyte effacement that is required for enteropathogenic Escherichia coli to increase tightjunction permeability encodes a chaperone for EspF. Infect Immun 2002;70:2271-2277.

19 Viswanathan VK, Lukic S, Koutsouris A, et al. Cytokeratin 18 interacts with the enteropathogenic Escherichia coli secreted protein F (EspF) and is redistributed after infection. Cell Microbiol 2004;6: 987-997.

20 McNamara BP, Donnenberg MS. A novel proline-rich protein, EspF, is secreted from enteropathogenic Escherichia coli via the type III export pathway. FEMS Microbiol Lett 1998;166:71-78.

21 Abe A, Heczko U, Hegele RG, et al. Two enteropathogenic Escherichia coli type III secreted proteins, EspA and EspB, are virulence factors. J Exp Med 1998;188: 1907-1916.

22 Deng W, Li Y, Vallance BA, et al. Locus of enterocyte effacement from Citrobacter rodentium: sequence analysis and evidence for horizontal transfer among attaching and effacing pathogens. Infect Immun 2001; 69:6323-6335.

23 Higgins LM, Frankel G, Douce G, et al. Citrobacter rodentium infection in mice elicits a mucosal Th1 cytokine response and lesions similar to those in murine inflammatory bowel disease. Infect Immun 1999;67:3031-3039.

24 Tauschek M, Strugnell RA, Robins-Browne RM. Characterization and evidence of mobilization of the LEE pathogenicity island of rabbit-specific strains of enteropathogenic Escherichia coli. Mol Microbiol 2002; 44:1533-1550.

25 Johnson E, Barthold SW. The ultrastructure of transmissible murine colonic hyperplasia. Am J Pathol 1979;97:291-313.

26 Savkovic SD, Villanueva J, Turner JR, et al. Mouse model of enteropathogenic Escherichia coli infection. Infect Immun 2005;73:1161-1170.

27 Wang F, Graham WV, Wang Y, et al. Interferon-gamma and tumor necrosis factor-alpha synergize to induce intestinal epithelial barrier dysfunction by up-regulating myosin light chain kinase expression. Am J Pathol 2005;166:409-419.
28 Ma TY, Boivin MA, Ye D, et al. Mechanism of TNF$\backslash\{$ alpha $\backslash\}$ modulation of Caco-2 intestinal epithelial tight junction barrier: role of myosin light-chain kinase protein expression. Am J Physiol Gastrointest Liver Physiol 2005;288:G422-G430.

29 Savkovic SD, Ramaswamy A, Koutsouris A, et al. EPEC-activated ERK1/2 participate in inflammatory response but not tight junction barrier disruption. Am J Physiol Gastrointest Liver Physiol 2001;281: G890-G898.

30 Muza-Moons MM, Koutsouris A, Hecht G. Disruption of cell polarity by enteropathogenic Escherichia coli enables basolateral membrane proteins to migrate apically and to potentiate physiological consequences. Infect Immun 2003;71:7069-7078.

31 Nagai T, Abe A, Sasakawa C. Targeting of enteropathogenic Escherichia coli EspF to host mitochondria is essential for the bacterial pathogenesis: critical role of the 16th leucine residue in EspF. J Biol Chem 2004; 280:2998-3011.

32 Nougayrede JP, Donnenberg MS. Enteropathogenic Escherichia coli EspF is targeted to mitochondria and is required to initiate the mitochondrial death pathway. Cell Microbiol 2004;6:1097-1111.

33 Heczko U, Carthy CM, O’Brien BA, et al. Decreased apoptosis in the ileum and ileal Peyer's patches: a feature after infection with rabbit enteropathogenic Escherichia coli O103. Infect Immun 2001;69:45804589.

34 Toivola DM, Krishnan S, Binder HJ, et al. Keratins modulate colonocyte electrolyte transport via protein mistargeting. J Cell Biol 2004;164:911-921.

35 Savkovic SD, Koutsouris A, Hecht G. PKC zeta participates in activation of inflammatory response induced by enteropathogenic E. coli. Am J Physiol Cell Physiol 2003;285:C512-C521.

36 Fish SM, Proujansky R, Reenstra WW. Synergistic effects of interferon gamma and tumour necrosis factor alpha on T84 cell function. Gut 1999;45:191-198.

37 Madara JL, Stafford J. Interferon-gamma directly affects barrier function of cultured intestinal epithelial monolayers. J Clin Invest 1989;83:724-727.

38 Youakim A, Ahdieh M. Interferon-gamma decreases barrier function in T84 cells by reducing ZO-1 levels and disrupting apical actin. Am J Physiol 1999;276: G1279-G1288.

39 Sugi K, Musch MW, Field M, et al. Inhibition of $\mathrm{Na}+, \mathrm{K}+$-ATPase by interferon gamma down-regulates intestinal epithelial transport and barrier function. Gastroenterology 2001;120:1393-1403.

40 Schmitz H, Fromm M, Bentzel CJ, et al. Tumor necrosis factor-alpha (TNFalpha) regulates the epithelial barrier in the human intestinal cell line HT-29/B6. J Cell Sci 1999;112(Part 1):137-146.

41 Rodriguez P, Heyman M, Candalh C, et al. Tumour necrosis factor-alpha induces morphological and functional alterations of intestinal HT29 cl.19A cell monolayers. Cytokine 1995;7:441-448.

42 Ma TY, Iwamoto GK, Hoa NT, et al. TNF-alpha-induced increase in intestinal epithelial tight junction permeability requires NF-kappa B activation. Am J Physiol Gastrointest Liver Physiol 2004;286:G367-G376.

43 Ramirez K, Huerta R, Oswald E, et al. Role of EspA and intimin in expression of proinflammatory cytokines from enterocytes and lymphocytes by rabbit enteropathogenic Escherichia coli-infected rabbits. Infect Immun 2005;73:103-113. 
44 Nash S, Stafford J, Madara JL. Effects of polymorphonuclear leukocyte transmigration on the barrier function of cultured intestinal epithelial monolayers. J Clin Invest 1987;80:1104-1113.

45 Parkos CA, Colgan SP, Delp C, et al. Neutrophil migration across a cultured epithelial monolayer elicits a biphasic resistance response representing sequential effects on transcellular and paracellular pathways. J Cell Biol 1992;117:757-764.

46 Colgan SP, Parkos CA, Delp C, et al. Neutrophil migration across cultured intestinal epithelial monolayers is modulated by epithelial exposure to IFNgamma in a highly polarized fashion. J Cell Biol 1993; 120:785-798.

47 Huber D, Balda MS, Matter K. Occludin modulates transepithelial migration of neutrophils. J Biol Chem 2000;275:5773-5778.
48 Kucharzik T, Walsh SV, Chen J, et al. Neutrophil transmigration in inflammatory bowel disease is associated with differential expression of epithelial intercellular junction proteins. Am J Pathol 2001;159: 2001-2009.

49 Zhou X, Giron JA, Torres AG, et al. Flagellin of enteropathogenic Escherichia coli stimulates interleukin-8 production in T84 cells. Infect Immun 2003;71: 2120-2129.

50 Ritchie JM, Waldor MK. The locus of enterocyte effacement-encoded effector proteins all promote enterohemorrhagic Escherichia coli pathogenicity in infant rabbits. Infect Immun 2005;73:1466-1474.

51 Blikslager AT, Roberts MC, Argenzio RA. Prostaglandin-induced recovery of barrier function in porcine ileum is triggered by chloride secretion. Am J Physiol 1999;276:G28-G36. 